\title{
Electrochemical Analysis of Shewanella oneidensis Engineered to Bind Gold Electrodes
}

\author{
A THESIS \\ SUBMITTED TO THE FACULTY OF THE GRADUATE SCHOOL \\ OF THE UNIVERSITY OF MINNESOTA \\ BY
}

Aunica L. Kane

IN PARTIAL FULFILLMENT OF THE REQUIREMENTS

FOR THE DEGREE OF

MASTER OF SCIENCE

Dr. Jeffrey A. Gralnick

March 2011 
(C) Aunica L. Kane 2011 


\section{Acknowledgements}

I would like to express my sincerest gratitude to my advisor Dr. Jeffrey Gralnick whose invaluable advice, suggestions, and mentorship made this thesis possible. I would also like to thank my committee members Dr. Daniel Bond and Dr. Christine Solomon for their support and helpful discussion along the way. Thanks also go to all of my lab mates in the Gralnick lab whose thoughtful conversations and dialogue have helped in forming this work.

Special thanks also go to my family. First and foremost, most thanks go to my husband Randall. None of my accomplishments thus far would have been possible without your love and encouragement. Through the ups and downs, you have consistently been my biggest supporter. To my parents, thank you for always supporting my dreams and never doubting I could reach the next step. Dad, thank you for fostering my love of science as a child with all of your crazy experiments. Mom, your love and support are what have helped me make it this far. Thank you all. 


\section{Dedication}

This thesis is dedicated along with a special thanks and deepest gratitude to Kenny and Tracy Lubke. Without your help my dream to go to college may have remained just a dream. I can never thank you enough for helping me through four long and expensive years of college. Thank you for being my 'second' family. 


\section{Table of Contents}

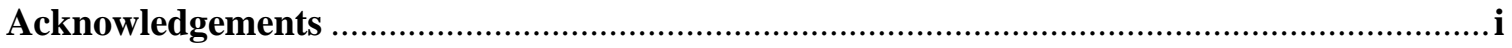

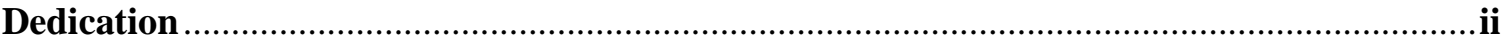

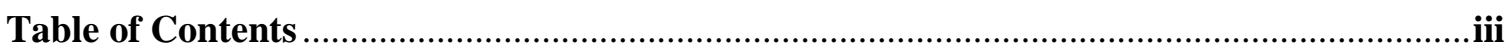

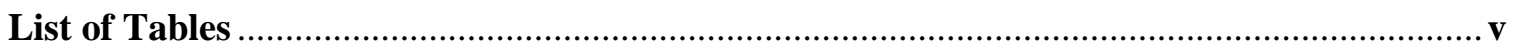

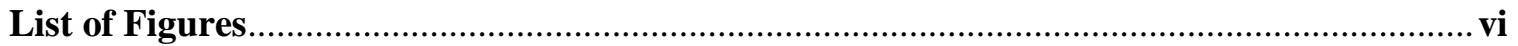

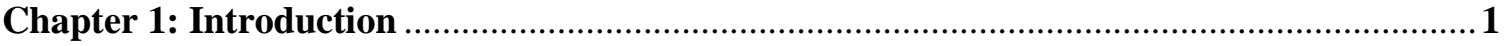

Chapter 2: Directing adhesion of Shewanella oneidensis to gold(111) via cell surface display

of a modified outer membrane porin, LamB, and a gold-binding polypeptide ....................... 3

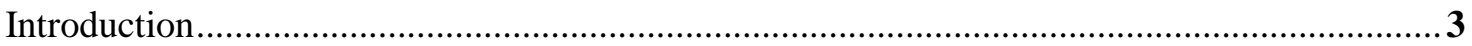

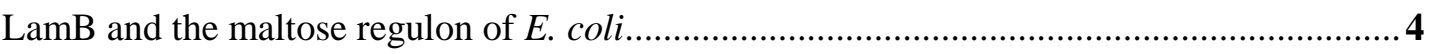

Surface Display and GBP-1 (Gold-Binding Peptide) ..........................................................

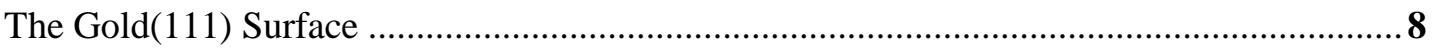

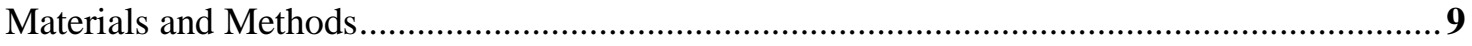

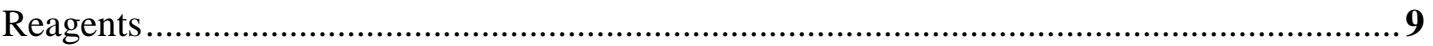

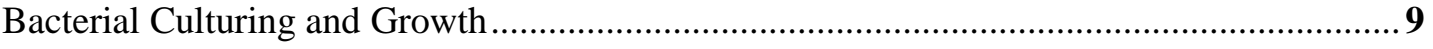

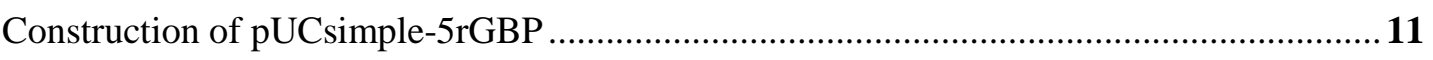

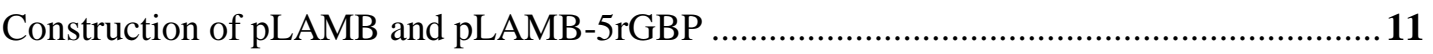

Growth Characterization with Arabinose ............................................................................ 12

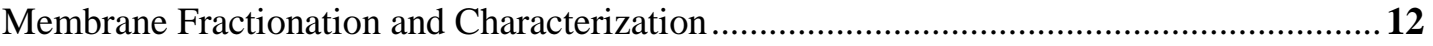

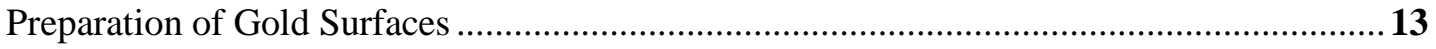

Characterization of Adhesion to Gold Surfaces................................................................. 13

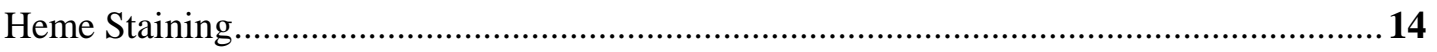

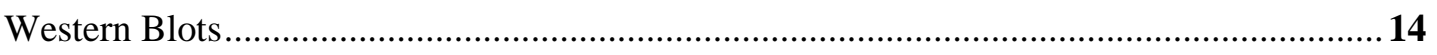

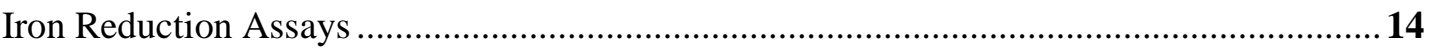




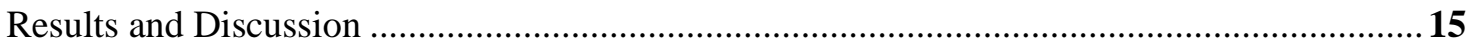

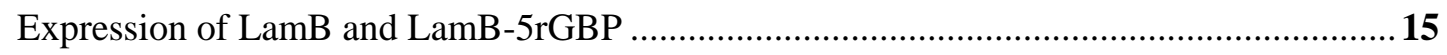

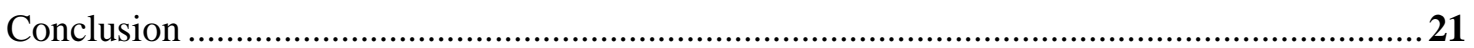

Chapter 3: Electrochemical Analysis of Shewanella Strains Engineered to Express a Gold-

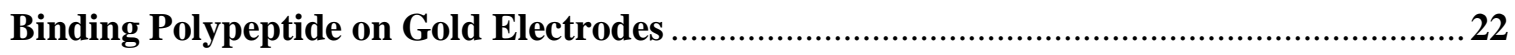

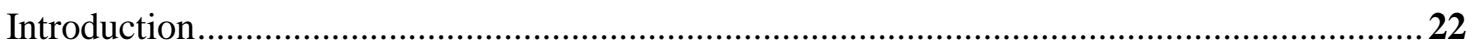

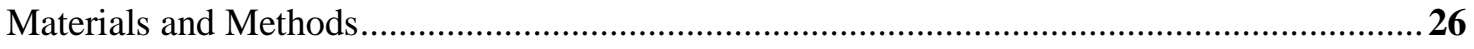

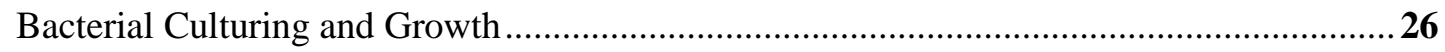

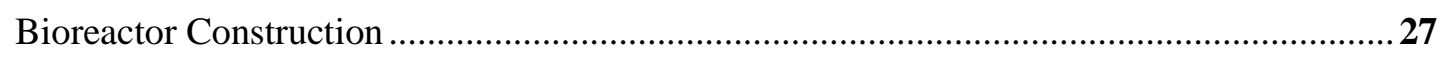

Current Analysis in Reactors Poised During Growth .....................................................27

Current Analysis in Reactors Not Poised During Growth ................................................28

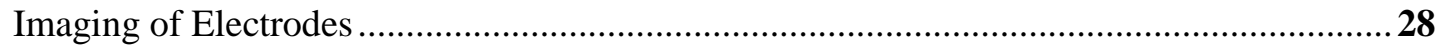

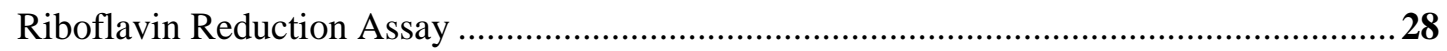

Reduction of $\mathrm{Au}(111)$ Electrodes By Riboflavin.............................................................29

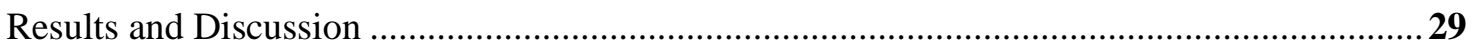

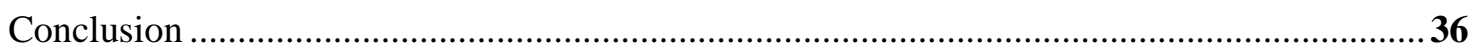

Chapter 4: Conclusions and Future Directions .............................................................. 38

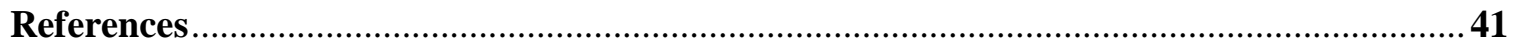




\section{List of Tables}

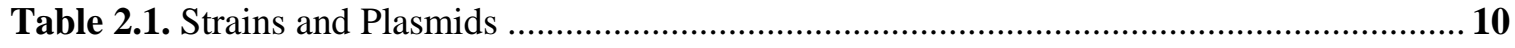

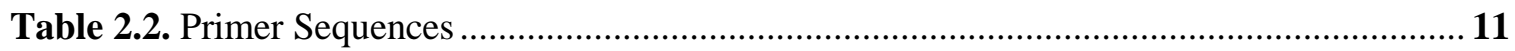

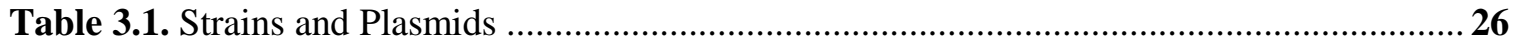




\section{List of Figures}

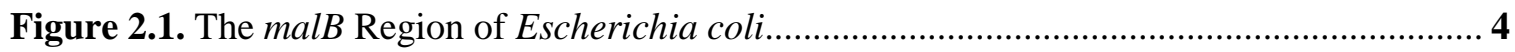

Figure 2.2. Schematics Based on the Crystal Structure of LamB .............................................5

Figure 2.3. Amino Acid Sequence and Characteristics of GBP-1 …....................................... 7

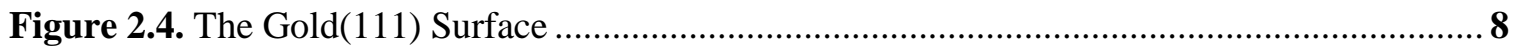

Figure 2.5. Membrane Fractions Subjected to SDS-PAGE .................................................. 16

Figure 2.6. Adhesion to Gold(111)-Plated Silicon Wafers ...................................................... 17

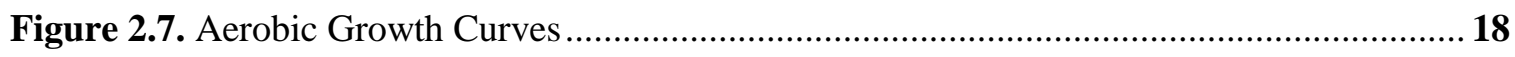

Figure 2.8. Outer Membrane Protein Heme Gel, Western Blot, and Color Change Image .......... 19

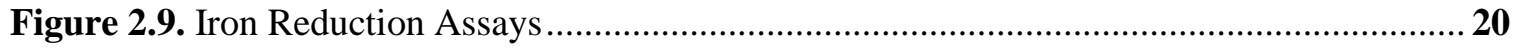

Figure 3.1. Three-Electrode Bioreactor Schematic .............................................................. 23

Figure 3.2. Chronoamperometry Analysis of Biofilm Formation on Poised Electrodes .............. 30

Figure 3.3. Riboflavin Reduction Assays ............................................................................ 31

Figure 3.4. Cyclic Voltammetry Schematic .................................................................. 32

Figure 3.5. Reduction of Gold Electrodes via Riboflavin ...................................................... 33

Figure 3.6. Chronoamperometry Analysis after Biofilm Formation on Un-Poised Electrodes .... 34

Figure 3.7. Images from Poised and Un-Poised Bioreactor Electrodes .......................................36 


\section{Chapter 1}

\section{Introduction}

Bio-electrochemical systems, which use microorganisms to catalyze redox reactions on an electrode, can be used to examine the mechanisms of electron flow from bacteria to naturallyoccurring insoluble electron acceptors. Interest in these systems and electrogenic bacteria has increased significantly in recent years. In bioreactor systems, bacteria oxidize a supplied carbon source serving as an electron donor and utilize an anodic electrode as the final acceptor. When operated in reverse, cathodic electrodes can also supply bacteria with the electrons needed to drive certain intracellular enzymatic reactions. Flow of these electrons through an external circuit allows for quantitative analyses of the processes involved in extracellular electron transport.

Model systems have been developed for use in bio-electrochemical systems, namely Shewanella and Geobacter species - this study will focus on the former. Shewanella are facultative anaerobes derived naturally from aquatic or subsurface environments at the oxic/anoxic interface (Hau and Gralnick, 2007). Survival in such environments requires the ability to utilize an array of final electron acceptors - a phenotypic hallmark of the Shewanella genus. Electrodes used in bio-electrochemical cells differ greatly at the molecular level from the substrates these bacteria encounter naturally; therefore, it is unlikely that these species have evolved specifically to interact with electrode surfaces. In fact, certain anode materials have shown to be incompatible for growth or attachment of Shewanella biofilms (Crittenden et al., 2006; Sun et al. 2010). Therefore, the basis of this work was to engineer a strain of Shewanella designed to bind electrode surfaces and improve whole-cell biocatalytic systems by encouraging microbe-electrode interactions and increasing overall current production.

Shewanella are gram negative members of the $\gamma$-Proteobacteria ubiquitously found in chemically-stratified aquatic and sedimentary environments (Fredrickson et al., 2008; Hau and Gralnick, 2007). This diverse distribution is made possible by the versatile respiratory capacity of Shewanella who are able to respire more than 20 organic and inorganic compounds in the absence of oxygen, many of which are insoluble or toxic metals (reviewed in Hau and Gralnick, 2007; Nealson and Scott, 2006). Examples of these include trimethylamine- $N$-oxide, fumarate, iron and 
manganese oxides, nitrate, uranium, and electrodes. Electron transfer to an insoluble acceptor requires a mechanism that enables the delivery of electrons from the cytosol to the extracellular substrate in a process termed extracellular respiration. Shewanella accomplishes this transfer of electrons using specialized membrane-bound and periplasmic electron carriers including quinones and $c$-type cytochromes which span the inner membrane, periplasm, and outer membrane. Their respiratory versatility is paralleled by the number of $c$-type cytochromes found within the Shewanella genome - over 40 have been identified to date (Heidelberg et al., 2002; Nealson and Scott, 2006).

Shewanella oneidensis MR-1 is the most widely studied strain in the genus, and many of the pathways required for extracellular respiration have been elucidated. With genetic manipulation techniques available and a sequenced genome, S. oneidensis has become an important model organism in biotechnological applications (Heidelberg et al., 2002; Kim et al., 2002; Marshall et al., 2006). Chapter 2 will begin with the engineering of MR-1 to adhere to gold(111) surfaces through the cell surface display of a modified Escherichia coli outer membrane porin, LamB, and an integrated gold-binding peptide, GPB-1. Surface display is a technique that fuses peptides of interest to native microbial components leading to their display on the outer surface of the cell. Briefly, lamB was amplified from E. coli K12 and modified to incorporate a repeating inorganic binding peptide, $g b p$ - 1 , into a permissive site within an outer loop of LamB. Transformed into S. oneidensis via an inducible plasmid, expression of this modified protein allowed for the adsorption of S. oneidensis to specific gold surfaces. Chapter 3 utilizes this engineered strain in three-electrode biorector systems to determine whether increased binding to gold electrode surfaces improves electrical output. A more in-depth background specific to each technique can be found in the introductory paragraphs of corresponding chapters. 


\section{Chapter 2}

\section{Directing adhesion of Shewanella oneidensis to gold(111) via cell surface display of a modified outer membrane porin, LamB, and a gold-binding polypeptide}

\section{Introduction}

Advances in recombinant DNA technologies, along with our increased understanding of microbial genomes and systems, have led to the generation of many powerful techniques in biotechnology. Through genetic engineering, we are now able to manipulate microorganisms to perform specific tasks. One application enabled by recombinant DNA technology is the display of heterologous peptides or proteins on the surface of microorganisms. Surface display is a technique that fuses peptides of interest to native microbial components leading to their display on the outer surface of the cell (reviewed in Lee et al., 2003; Rutherford and Mourez, 2006). The first successful use of surface display was reported in 1985 by George Smith whose lab generated a fusion phage by expressing a cloned antigen within the pIII protein (Smith, 1985). This foreign sequence was displayed on the phage surface in an immunologically accessible form without disrupting pIII function. This expression system was limited in the size of peptides that could be expressed; therefore, development of bacterial display systems soon followed.

LamB and OmpA from Escherichia coli were the first outer membrane proteins utilized in bacterial cell surface display (Charbit et al., 1986; Freudl et al., 1986). LamB, the protein used further in this study, is an integral outer membrane protein that aides in maltose and maltodextrin uptake (Szmelcman and Hofnung, 1975). OmpA is mainly important in cell structural integrity but also serves as a receptor for multiple phage and is required in F-conjugation (Wang, 2002). Before the crystal structure for LamB became available, Charbit and colleagues attempted to gain insight into the overall structure of LamB by inserting the C3 epitope from the VP1 coat protein of poliovirus into permissive sites and probing for extracellular regions. This fusion led to expression of the heterologous protein and hence allowed probing for LamB regions located on the outside of the cell by $\mathrm{C} 3$-specific antibodies. Although this method was originally designed to 
gain information on the structure of LamB, the authors also noted that it held potential to be of use in generating larger fusions.

\section{LamB and the maltose regulon of $E$. coli}

LamB, also known as maltoporin, is an integral outer membrane protein encoded by a gene located in the malB region of the E. coli genome. This region consists of two divergently transcribed operons, malEFG and malK-lamB-malM, which encode for the maltose and maltodextrin uptake components (Fig. 2.1). LamB facilitates the transport of maltose and maltooligosaccharides across the outer membrane while the membrane transporter $\mathrm{MalFGK}_{2}$ uses ATP to drive active transport across the inner membrane (Szmelcman and Hofnung, 1975; reviewed in Boos and Shuman, 1998). $\mathrm{MalFGK}_{2}$ is an $\mathrm{ABC}$ transporter composed of two integral membrane proteins, MalF and MalG, and two copies of the ATP-hydrolyzing unit, MalK. MalE, also known as maltose binding protein (MBP), is located in the periplasm where it binds maltose and maltodextrins and delivers them to the transporter. It consists of 2 nearly symmetrical lobes which interact with the integral membrane proteins MalF and MalG (Hor and Shuman, 1993). MalM is a periplasmic protein of unknown function (Gilson et al., 1986).

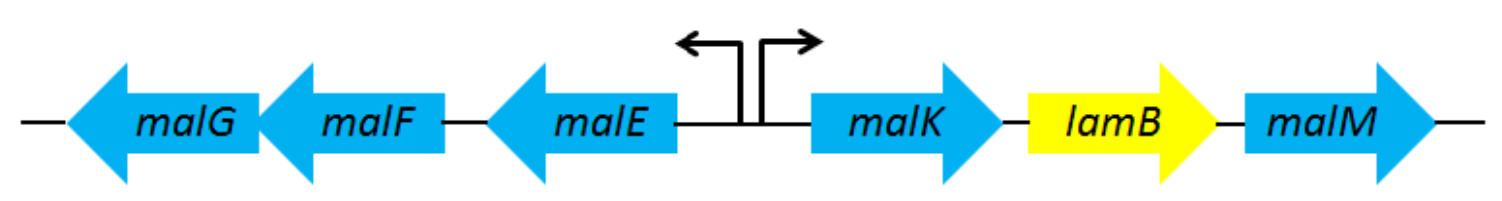

Figure 2.1: The malB region of Escherichia coli. This region consists of two divergently transcribed operons, malEFG and malK-lamB-malM, positively regulated by the activator MalT.

The maltose regulon is a positively controlled system. The activator, MalT, is induced by internal maltotriose and ATP, and its expression also requires the presence of the cAMP/CAP complex (reviewed in Boos and Shuman, 1998). Certain mutations within malT have been identified that lead to the constitutive expression all mal genes (Debarbouille et al., 1978). MalK also plays a role in regulation acting as a repressor by interacting with MalT and inactivating it in the absence of maltose. When maltose is present, it is recognized by MalE and transported to the $\mathrm{MalFGK}_{2}$ transporter complex which signals MalK to hydrolyze its bound ATP thereby eliminating its affinity for MalT (reviewed in Boos and Shuman, 1998). 
LamB, a member of the sugar porin family, is responsible for the transport of maltose and maltodextrins across the outer membrane but was first discovered, and hence named, as the receptor for bacteriophage lambda (Randall-Hazelbauer and Schwartz, 1973). In its active form, LamB is a trimer composed of three 421 aa monomers with each subunit forming a channel of 18 antiparallel $\beta$-strands (Clement and Hofnung, 1981; Schirmer et al., 1995). Schematics based on the crystal structure are outlined in Figure 2.2. A series of aromatic residues termed the "greasy slide" line each channel in an arrangement most likely important for sugar binding (Schirmer et al., 1995). The overall structure of the barrel is characterized by long irregular loops on the extracellular side and short turns in the periplasm. Loops that protrude on the outside of the cell are numbered as outlined in Figure 2.2. Loops 1, 3, and 6 fold inside the channel with loop 3 constricting pore size. The second loop is responsible for attachment to the adjacent subunit in the trimer. On the extracellular face, loops 4, 6, and 9 form large protrusions and are thought to attract maltodextrins (Newton et al., 1996; Schirmer et al., 1995).

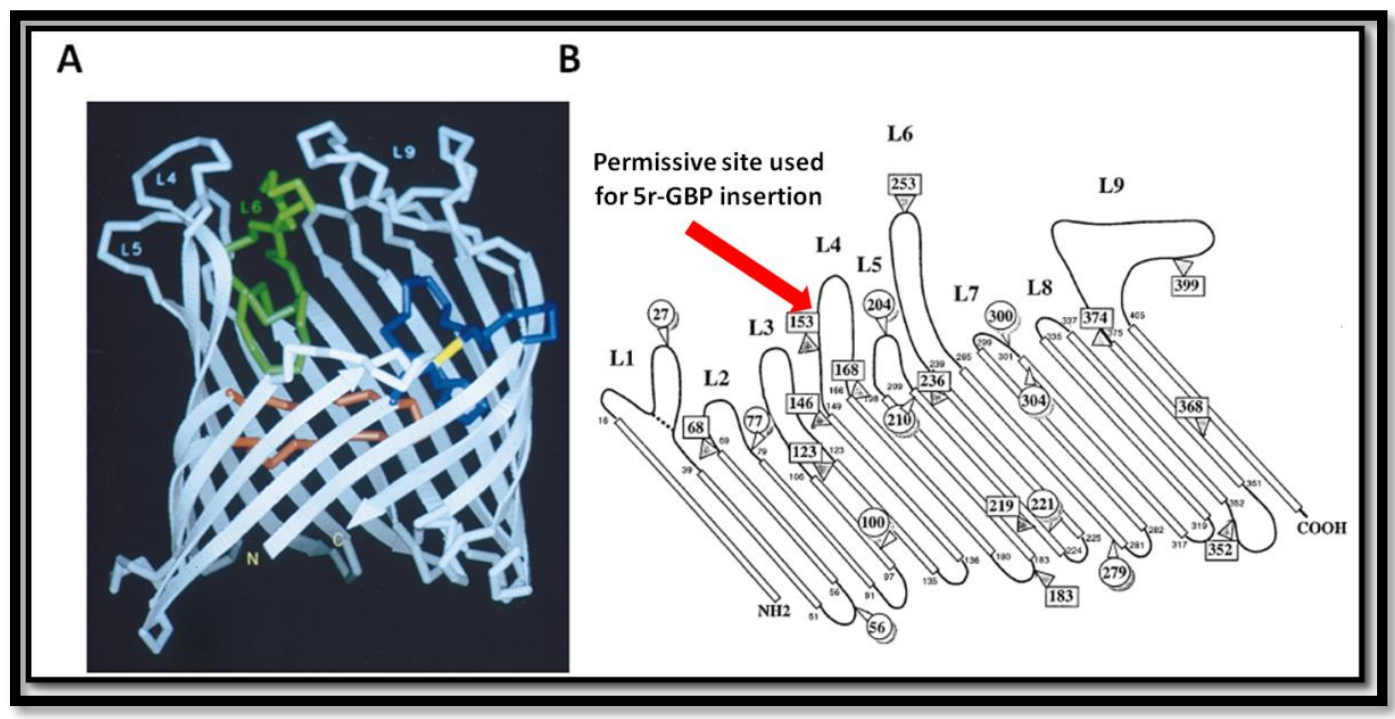

Figure 2.2: (A) 3-dimentional schematic from the crystal structure of LamB (Schirmer et al., 1995). (B) 2dimentional adaptation of LamB and the extracellular numbered loops (Newton et al., 1996). The red arrow indicates the permissive region used for 5 repeats of gold-binding peptide insertion.

These outer loops have been the focus of research utilizing surface display applications due to the presence of 'permissive sites' within these regions of the protein. Permissive sites allow for the insertion of foreign amino acids without affecting folding, transport, or function of the native protein and were originally identified by a group studying the topology of LamB (Boulain et al., 1986). The Hofnung laboratory developed a method to probe the arrangement of 
LamB within the membrane before the X-ray structure became available by inserting a reporter epitope into various sites within the protein and probing those sites using a monoclonal antibody (Charbit et al., 1986). Although they developed this method to obtain structural information, they also discussed the idea that such an approach could potentially be useful in generating larger hybrid proteins. In this current study, a permissive site located within loop 4 at amino acid position 153 was used to create a hybrid protein containing a gold-binding polypeptide which conferred the ability for Shewanella to bind gold(111) surfaces (Figure 2.2).

\section{Surface Display and GBP-1 (Gold-Binding Peptide)}

Interactions between proteins and inorganic surfaces play important roles in everything from technological applications to natural biological processes. Therefore, research in many labs has centered on defining the properties that govern the recognition of surfaces by specific proteins. In these approaches, research aimed at uncovering the mechanisms that drive specific protein-surface recognition has also led to the generation of multiple inorganic binding peptides. By scanning large libraries of randomized peptides, one can gain insight into the recognition properties of specific sequences.

Combinatorial biology techniques such as phage and cell surface display have been used to select for peptide sequences that preferentially bind specific targets (reviewed in Sarikaya et al., 2003). Using these approaches, large libraries of random peptides are displayed as heterologous molecules on the bacterial or phage surface by incorporation of peptide sequences into permissive sites within outer membrane, flagella, or phage coat proteins. Recombinant cells or phage are then exposed to the target surface, and those that bind are recovered. Multiple rounds of selection are performed to select for the tightest binders. Surface display is a powerful technique since it allows the user to directly connect the phenotype-binding to the surface, to the genotype - the binding peptide sequence.

Gold-binding peptide (GBP-1) was originally selected from a cell-surface display library of approximately 5 million polypeptides displayed on the outer surface of the E. coli outer membrane protein LamB (Brown, 1997). Multiple peptides with affinity to gold were uncovered in this study, and GBP-1(sequence MHGKTQATSGTIQS) was the best binder. No consensus sequence for gold-binding was clear from the multiple gold-binding peptides discovered, but all 
were rich in the amino acids serine and threonine. This study also found that by polymerizing or repeating gold-binding sequence units, one could further increase binding affinity towards gold.

While binding of most peptides and self-assembled monolayers to gold surfaces tends to involve thiol linkages (Fenter et al., 1994; Ulman, 1996), the affinity for gold-binding peptides appears to be different since none contain cysteine. Much work has been undertaken recently to uncover the physiochemical principles responsible for this binding. Molecular dynamics studies looking at individual amino acid interactions with the gold(111) surface outlined the following trend for gold affinity: aromatic > sulfur containing > positive > polar > aliphatic > negative, and noted that His and Met could create interactions with gold approaching covalent bonds (Hoefling et al., 2010a). This trend predicts low affinity for gold by all but the first few amino acids of GBP-1 (Fig. 2.3).

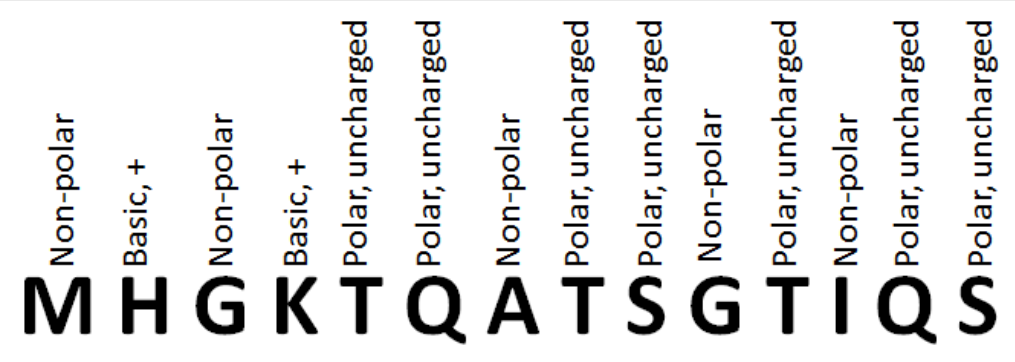

Figure 2.3. Amino acid sequence and characteristics of GBP-1.

Other studies, using various gold-binding peptides and molecular simulations with homopolypeptides suggest that individual amino acid characteristics cannot fully explain peptide adsorption to gold surfaces, but that overall peptide organization and structure also contribute (Heinz et al., 2009; Verde et al 2009). These studies found that strong gold binders included Arg, Asp, Asn, His, Ile, and Tyr, and that strong adsorption was also seen by less attractive residues Gly, Thr, and Ser. Strong binders also tended to exhibit a flat, 2-dimentional conformation such that most residues were in direct contact with the gold surface. Hence, it is possible to envision that the peptide sequence, or the arrangement of amino acids, plays a larger role than individual amino acid affinity for binding to gold surfaces. In fact, having less strongly binding peptides near strong binders increased direct contact and binding overall (Heinz et al., 2009). 
Protein flexibility, or the lack of rigid secondary structure, also seems to contribute to overall gold peptide binding — strong binding is not only due to the presence of high affinity binders, but is also due to the arrangement of those amino acids so they may be in contact with the gold surface. Research indicates that gold surfaces may lead to unfolding of native proteins, so $\beta$-sheets or naturally unfolded peptides may be the best gold binders (Hoefling et al., 2010b). Verde et al (2009) conducted an experiment to probe the role of secondary structure in goldbinding using two peptides containing equal amounts of "strong binding" amino acids, but that exhibited opposite behavior on gold. The non gold-binding peptide (6NGBP) was predicted to contain mostly helices, while 6 repeats of GBP-1 (6GBP) was predicted to be mainly random coil with only $25 \%$ of amino acids in either helices or $\beta$-sheets.

\section{The Gold(111) Surface}

Cell surface display systems select for peptides that bind specific surfaces based on their physical and chemical properties, and GBP-1 selectively binds the gold(111) surface (Brown, 1997; Tamerler et al., 2006). Gold(111) is a single crystal, atomically flat surface and is the most densely packed form of crystalline gold (Carabineiro and Nieuwenhuys, 2009). Images of this surface are provided in Figure 2.4 and display the common 'herringbone' structure characteristic of gold(111). The numbers within the parentheses are Miller indices which describe the orientation of atomic planes within the crystal lattice.

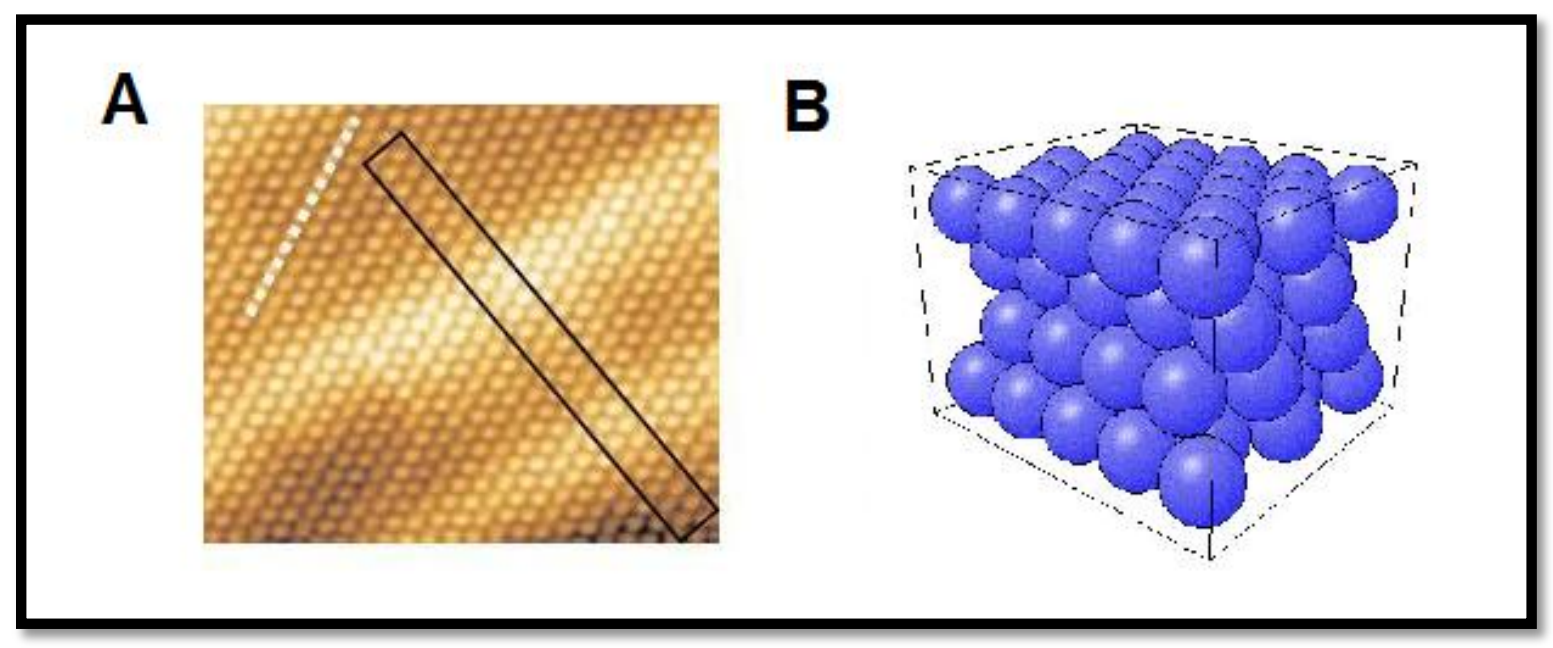

Figure 2.4. The gold(111) surface. A) Scanning tunneling image of the single crystal gold(111) surface displaying the characteristic 'herringbone' pattern (Schouteden et al., 2009) B) Schematic of the gold(111) surface generated using Surface Explorer (http://w3.rz-berlin.mpg.de/ rammer/surfexp_prod/SXinput.html) 
In the current study, surface display was utilized to express LamB modified to incorporate five repeating units of GBP-1 (5rGBP) on the outer membrane of Shewanella oneidensis to improve the attachment of engineered cells to gold surfaces. Gold was chosen as the target surface due to the second goal of this research - increasing current production in goldelectrode bioreactors (Chapter 3). Gold is an attractive source as an anode material in bioreactors due to its high conductivity and high signal-to-noise ratio in data collection, yet previous research has shown that Shewanella is resistant to colonization on gold surfaces (Crittenden et al., 2006; Sun et al, 2010). The inability to couple with gold indicated that cells were either unable to interact with gold or that the surface itself was toxic. It is possible that strong adsorption on gold denatures electron transfer proteins such as outer membrane cytochromes; yet on the other hand, inability to interact and couple with gold could be due to poor binding (Chen et al, 2002). Electron transfer rates are exponentially proportional to the distance between the cytochrome heme group and the electrode surface, and these rates decrease as the distance between the heme group and the surface of the electron acceptor increases (Chen et al., 2002).

\section{Materials and Methods}

\section{Reagents}

Restriction enzymes, T4 DNA ligase, and Antarctic phosphatase were obtained from New England Biolabs (Ipswich, MA). $P f x 50^{\mathrm{TM}}$ high fidelity polymerase and $\mathrm{pCR} ®$-BluntII-TOPO® kits were acquired from Invitrogen (Carlsbad, CA). The QuikChange ${ }^{\mathrm{TM}}$ kit was provided by Stratagene (now Agilent, Santa Clara, CA). All related reactions were carried out according to these manufacturers' instructions. Kits for gel purification and plasmid mini preps were purchased from Qiagen (Valencia, CA).

\section{Bacterial Culturing and Growth}

All strains and plasmids used in this study are listed below in Table 2.1. Single colonies from a freshly streaked $-80^{\circ} \mathrm{C}$ stock were used to inoculate overnight cultures. These cultures were grown for 16 hours in Luria-Bertania (LB) medium supplemented with $50 \mu \mathrm{g} / \mathrm{mL}$ kanamycin $(\mathrm{Km})$ to support the maintenance of plasmids. Unless specified otherwise, cultures were shaken continuously at $250 \mathrm{rpm}$ and incubated at $30^{\circ} \mathrm{C}$ or $37^{\circ} \mathrm{C}$ for Shewanella or E. coli strains, respectively. Shewanella Basal Medium (SBM) pH 7.2 was used where specified and contained, per 1 liter of medium, $0.225 \mathrm{~g} \mathrm{~K}_{2} \mathrm{HPO}_{4}, 0.225 \mathrm{~g} \mathrm{KH}_{2} \mathrm{PO}_{4}, 0.46 \mathrm{~g} \mathrm{NaCl}, 0.225 \mathrm{~g}\left(\mathrm{NH}_{4}\right)_{2} \mathrm{SO}_{4}, 0.117$ 
$\mathrm{g} \mathrm{MgSO}_{4} \cdot 7 \mathrm{H} 2 \mathrm{O}, 100 \mathrm{mM}$ HEPES buffer. This medium was supplemented with $25 \mu \mathrm{g} / \mathrm{mL} \mathrm{Km}$, vitamin and mineral mix, and $0.05 \%$ casamino acids as described previously (Hau et al., 2008).

\begin{tabular}{|c|c|c|}
\hline Strain or Plasmid & Genotype/Characteristics & Reference/Source \\
\hline S. oneidensis strain MR-1 & Isolated from L. Oneida, NY & $\begin{array}{l}\text { Venkateswaran et } \\
\text { al., } 1999\end{array}$ \\
\hline $\begin{array}{l}\text { E. coli K12 strain } \\
\text { MG1655 }\end{array}$ & Wild Type & $\begin{array}{l}\text { Kindly provided by } \\
\text { Dr. Arkady } \\
\text { Khodursky at the } \\
\text { University of } \\
\text { Minnesota }\end{array}$ \\
\hline E. coli strain UQ950 & $\begin{array}{l}\text { DH5 } \alpha \text { host for cloning: F- } \arg F- \\
\text { lac) } 169 \text { 80dlacZ58(M15) glnV44(AS) } \\
\text { rfbD1 gyrA96(NalR) recAl endA1 } \\
\text { spoT1 thi-1 hsdR17 deoR pirt }\end{array}$ & $\begin{array}{l}\text { Saltikov and } \\
\text { Newman, } 2003\end{array}$ \\
\hline E. coli strain WM3064 & $\begin{array}{l}\text { DAP auxotroph used for conjugation: } \\
\text { thrB1004 pro thi rpsL hsdS lacZM15 } \\
\text { RP4-1360 (araBAD)567 } \\
\text { dapA1341::[erm pir }(w t)]\end{array}$ & $\begin{array}{l}\text { Saltikov and } \\
\text { Newman, } 2003\end{array}$ \\
\hline JG1301 & $\begin{array}{l}\text { S. oneidensis MR-1: empty } \\
\text { pBBAD } 18 \mathrm{~K} \text { vector }\end{array}$ & This Study \\
\hline JG1302 & S. oneidensis MR-1: lamB & This Study \\
\hline JG1303 & S. oneidensis MR-1: lamB-5rGBP & This Study \\
\hline pBBAD18K & $\begin{array}{l}7.6 \mathrm{~kb} \text { broad host range cloning vector; } \\
\text { L-arabinose induced; } \mathrm{Km}^{\mathrm{R}}\end{array}$ & $\begin{array}{l}\text { Sukchawalit et al., } \\
1999\end{array}$ \\
\hline pUC57simple-5rGBP & $\begin{array}{l}\text { pUC57simple containing } 5 \text { r-gbp (gold- } \\
\text { binding peptide) synthesized by } \\
\text { GenScript }\end{array}$ & $\begin{array}{l}\text { GenScript } \\
\text { (Piscataway, NJ) }\end{array}$ \\
\hline
\end{tabular}


plamB

plamB-5rGBP
pBBAD18K containing lamB (cloned from E. coli K12 strain MG1655)

modified at codons $155-157$ to introduce PstI and XhoI restriction sites

plamB containing $5 r-g b p$ from pUCsimple-5rGBP
This Study

\author{
plamb-5rGBP
}

This Study

Table 2.1. Strains and plasmids used in this study.

\section{Primer}

LamB-Fwd

LamB-Rev

LamB-mod

LamB-mod-anti 5'-nncgagctcatccggcctacatttgacag-3'

5'-gatatttctggtcctggtgccggtctgcagctcgagga tgttggcttcggtaaactctctctg-3'

5'-cagagagagtttaccgaagccaacatcctcgagctgcagaccggcaccaggaccagaaata tc- 3 '

Table 2.2 Primer sequences used in this study.

\title{
Construction of pUCsimple-5rGBP
}

The sequence for 5r-gbp, five repeating units of $g b p l$ (Brown, 1997) (MHGKTQATSGTIQS) flanked with PstI and XhoI restriction sites, was synthesized by GenScript (Piscataway, NJ) and cloned into pUCsimple.

\section{Construction of pLAMB and pLAMB-5rGBP}

Primers used in the construction of plasmids are listed in Table 2.2. LamB was amplified using genomic DNA from E. coli K12 strain MG1655 as the PCR template and primers LamB-Fwd and LamB-Rev. This PCR fragment was then cloned into $\mathrm{pCR} \circledast$-BluntII-TOPO $®$ and sequenced. After restriction enzyme digests of the TOPO vector with ApaI and SacI, the fragment was gel purified and ligated into the corresponding sites of the phosphatase treated and gel purified 
pBBR1mcs-5. LamB was then modified at codons 154-157, utilizing the QuikChange ${ }^{\mathrm{TM}}$ kit and primers LamB-mod and LamB-mod-anti, to introduce PstI and XhoI sites to allow for the future incorporation of $5 \mathrm{r}-g b p$. The $5 \mathrm{r}-g b p$ fragment was excised from pUCsimple-5rGBP with PstI and XhoI. This fragment was then gel purified and ligated into the corresponding sites of pLAMB-5 to create pLAMBGBP-5.

The constitutive expression of LamB in the pBBR1-mcs5 vector was toxic in both $E$. coli and $S$. oneidensis, resulting in small colony size and the release of an insoluble white substance in liquid overnight culture medium. The plasmid pLAMB-5 was unstable in E. coli, and sequencing results often indicated base deletions and additions that altered the reading frame of $\operatorname{lam} B$. The addition of $5 \mathrm{r}-\mathrm{gbp}$ further compounded this instability. To overcome these issues, lamB and lamB-5rgbp were transferred to the arabinose-inducible vector pBBAD18K. Primers LamB-Fwd and LamBRev were used to re-amplify the lamB-5rgbp sequence from purified and sequenced pLAMB-5 and pLAMBGBP-5 by the high-fidelity polymerase $P f x 50^{\mathrm{TM}}$. This fragment was cloned into

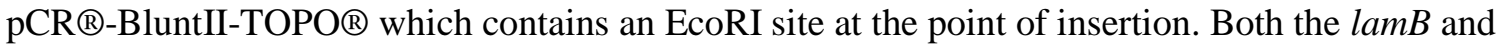
lamB-5rgbp sequences were then digested via SacI and EcoRI, gel purified, and ligated into the corresponding sites of $\mathrm{pBBAD} 18 \mathrm{~K}$. This generated the final plasmids utilized in the rest of this study: pLAMB and pLAMB-5rGBP.

\section{Growth Characterization with Arabinose}

Cells were inoculated to an $\mathrm{OD}_{600 \mathrm{~nm}} \sim 0.08$ in triplicate from an aerobic LB overnight culture and supplemented with $50 \mu \mathrm{g} / \mathrm{mL} \mathrm{Km}$. At an $\mathrm{OD}_{600 \mathrm{~nm}} \sim 0.5$, cultures were induced with $0.05 \% \mathrm{~L}$ arabinose. Time points were taken periodically to track growth of these cultures.

\section{Membrane Fractionation and Characterization}

One liter of LB medium, supplemented with $20 \mathrm{mM}$ lactate and $40 \mathrm{mM}$ fumarate, was inoculated with $10 \mathrm{~mL}$ of an aerobic LB overnight culture. These cultures were induced at an $\mathrm{OD}_{600 \mathrm{~nm}} \sim 0.5$ with $0.05 \%$ L-arabinose. After a 2.5 -hour induction period, cells were harvested by centrifugation for 10 minutes at $10,000 \mathrm{rpm}$. The following steps were all done at $4^{\circ} \mathrm{C}$. Pellets were washed in $10 \mathrm{mM}$ Tris-HCl, pH 8.0 with $1 \mathrm{mM}$ EDTA and $10 \mu \mathrm{M}$ PMSF and centrifuged again at $12,000 \mathrm{x}$ $\mathrm{g}$ for 10 minutes. These cells were resuspended in $40 \mathrm{~mL}$ of $10 \mathrm{mM}$ Tris-HCl, $\mathrm{pH} 7.5$ containing $10 \mu \mathrm{M}$ PMSF and then passed three times through a French press at 15,000 psi. To remove cell debris and unlysed cells, the suspension was centrifuged at $12,000 \mathrm{x}$ g for 20 minutes. The 
resulting supernatant was then centrifuged for an hour at $100,000 \mathrm{x} g$ to separate the soluble fraction from the crude membrane pellet. This pellet, which contained the total membrane, was resuspended in $1 \mathrm{~mL}$ of $10 \mathrm{mM}$ HEPES and added to a 10\% step sucrose gradient (35\% - 55\%). These were spun at 25,000 $\mathrm{x}$ g for 17 hours resulting in the separation of the outer and inner membranes. Aliquots of bands corresponding to each membrane were collected, pooled and dialysed in $10 \mathrm{mM}$ HEPES, pH 7.5 overnight with one exchange of buffer. These samples were then concentrated using Millipore columns, and a BCA assay was performed to determine the resulting protein concentrations.

To visualize cellular localization of LamB and LamB-5rGBP, membrane fractions were separated on a 4-12\% Bis-Tris gel subjected to SDS-polyacrylamide gel electrophoresis. For both the cytoplasmic and outer membrane fractions, $8 \mu \mathrm{g}$ of total protein was added to each lane. The resulting gel was visualized by staining with Comassie.

\section{Preparation of Gold Surfaces}

A gold(111)-coated silicon oxide wafer was purchased from Sigma and was cut into 1" x 1/4" pieces utilizing an ultrasonic mulling machine at the University of Minnesota's Electrical Engineering Machine Shop. Prior to cleaning with piranha solution, gold pieces were placed in acetone overnight followed by ethanol for one hour to remove all organic material. The gold was then placed into a 3:1 (conc. sulfuric acid: 30\% hydrogen peroxide) piranha solution for $20 \mathrm{~min}$ and washed three times in filtered and autoclaved $\mathrm{ddH}_{2} \mathrm{O}$. These surfaces were then dried with filtered $\mathrm{N}_{2}$ gas and used immediately.

\section{Characterization of Adhesion to Gold Surfaces}

Cells were inoculated to an $\mathrm{OD}_{600 \mathrm{~nm}} \sim 0.08$ from an aerobic $\mathrm{LB}$ overnight culture and supplemented with $50 \mu \mathrm{g} / \mathrm{mL} \mathrm{Km}$. At an $\mathrm{OD}_{600 \mathrm{~nm}} \sim 0.5$, cultures were induced with $0.05 \% \mathrm{~L}$ arabinose. Freshly piranha-cleaned gold was added when cells reached stationary phase $\left(\mathrm{OD}_{600 \mathrm{~nm}}\right.$ $=1.0$ ). Tubes containing the cultures and gold were then secured at an angle, allowing the entire gold surface to be in solution, and left to sit on the bench top for 16 hours. Following this overnight incubation, the gold wafers were removed from the tubes and rinsed three times in PBS. To ensure the removal of all unbound cells, gold pieces were placed in SBM and washed for 1 minute on a rocker (Boekel Scientific, Feasterville, PA). 
Cells bound to the gold surface were stained using the LIVE/DEAD BacLight ${ }^{\mathrm{TM}}$ Bacterial Viability Kit (Kit L7012) from Invitrogen according to the manufacturer's instructions.

Fluorescence was then visualized using an Olympus IX70 inverted fluorescent microscope and analyzed utilizing the SPOT ${ }^{\mathrm{TM}}$ Basic software. Six images were taken of each surface, and representative images were chosen.

\section{Heme Staining}

Cultures $(5 \mathrm{~mL}$ ) were inoculated in LB supplemented with $50 \mu \mathrm{g} / \mathrm{mL} \mathrm{Km}$ from an aerobic overnight culture to an $\mathrm{OD}_{600 \mathrm{~nm}} \sim 0.08$ and were induced with $0.05 \% \mathrm{~L}$-arabinose at mid-log phase. After a 2.5-hour induction period, cells had reached stationary phase and were spun down and resuspended in $500 \mu \mathrm{L}$ of $10 \mathrm{mM}$ HEPES buffered at $\mathrm{pH}$ 7.5. Samples were then sonicated to lyse cells and subsequently centrifuged to clear cell debris. Total protein from these samples was quantified using a BCA assay. Standard SDS loading buffer without a reducing agent was added and each sample was heated for 5 minutes at $80^{\circ} \mathrm{C}$. Total protein $(10 \mu \mathrm{g})$ was then added to individual wells of a 4-12\% bis-tris gradient gel, and heme-containing proteins were visualized due to the peroxidase activity of cytochromes using a staining technique outlined in Thomas et al (1976). Briefly, gels were placed in a $30: 706.3 \mathrm{mM}$ tetramethylbenzidine: $0.25 \mathrm{M}$ sodium acetate solution pH 5.0 for 2 hours in the dark after which bands were visualized by adding $30 \mathrm{mM}$ hydrogen peroxide.

\section{Western Blots}

Membrane fractions were collected as described previously and $8 \mu \mathrm{g}$ of total protein was added to individual wells of a $12 \%$ bis-tris gel. The gel was then transferred to a PVDF membrane for Western blot analysis using an antibody against MtrB. Addition of the secondary antibody and the developing process were carried out according to the manufacturer's instructions (Pierce).

\section{Iron Reduction Assays}

LB cultures $(2 \mathrm{~mL})$ were inoculated to an initial $\mathrm{OD}_{600 \mathrm{~nm}} \sim 0.08$ from an aerobic $\mathrm{LB}$ overnight culture and supplemented with $50 \mu \mathrm{g} / \mathrm{mL} \mathrm{Km}$. After a 2.5-hour induction period, cells were washed and resuspended to an $\mathrm{OD}_{600 \mathrm{~nm}}=0.4$ or 1.8 in $\mathrm{SBM}$ for iron citrate and iron oxide experiments, respectively. $30 \mu \mathrm{L}$ of this suspension was added to $270 \mu \mathrm{L}$ of SBM supplemented with $10 \mathrm{mM}$ HEPES, $20 \mathrm{mM}$ lactate, and $10 \mathrm{mM}$ iron in a 96 well plate. Samples were degassed for 15 minutes and kept anaerobic in a GasPak System petri dish container. At each time point, 50 
$\mu \mathrm{L}$ of $5 \mathrm{~N} \mathrm{HCl}$ was added to corresponding wells to prevent the oxidation of $\mathrm{Fe}(\mathrm{II})$, and $30 \mu \mathrm{L}$ of this suspension was added resulting in a $1: 10$ dilution into $0.5 \mathrm{~N} \mathrm{HCl}$ to yield $\mathrm{Fe}(\mathrm{II})$ concentrations within the range of standard curves. $50 \mu \mathrm{L}$ of the $1: 10$ cell/ $0.5 \mathrm{~N} \mathrm{HCl}$ mix was then added to another plate containing $300 \mu \mathrm{L}$ ferrozine reagent (Stooky, 1970) and read immediately at $562 \mathrm{~nm}$. The amount of Fe(II) produced was extrapolated from a standard curve.

\section{Results and Discussion}

\section{Expression of LamB and LamB-5rGBP}

Gold surfaces differ greatly, both chemically and physically, at the molecular level compared to substrates that Shewanella species encounter naturally. Additionally, previous research has indicated that the gold surface is resistant to colonization by Shewanella (Crittenden et al., 2006; Sun et al., 2010). This could indicate either that these species are unable to bind and utilize gold surfaces, or that the surface itself is toxic. As to the later, it is interesting to note that Geobacter sulfurreducens is able to colonize and use gold surfaces as a final electron acceptor in both fuel cells and bioreactors. In fuel cell studies, Geobacter biofilms were heterogeneous on gold and detached easily from the surface compared to biofilms on graphite (Richter et al., 2008). Although the amount of current per gram cell protein was comparable for gold and graphite, current density was 4-fold less in fuel cells with G. sulfurreducens on gold and was attributed to less biomass on the surface (Richter et al., 2008). Robust G. sulfurreducens biofilms have been produced in bioreactors (Liu et al., 2010). In these reactors, current density increased when gold line array electrodes were used which, due to the spacing, allowed more biomass to colonize the electrode surface compared to rectangular gold electrodes (Liu et al., 2010). Therefore it is reasonable to believe that engineering strains with increased affinity for gold could overcome the recalcitrance of Shewanella for colonization of gold surfaces.

The E. coli outer membrane protein LamB and the fusion protein LamB-5rGBP were successfully displayed on the outer surface of $S$. oneidensis. Outer and cytoplasmic membrane fractions were prepared from both un-induced and induced cultures of the empty vector control, LamB, and LamB-5rGBP expressing strains. These fractions were then subjected to SDS polyacrylamide gel electrophoresis (Figure 2.5). Expression of recombinant proteins was only seen in the outer membrane fractions of arabinose-induced cultures (bold type). An extra band was observed in the induced LamB-5rGBP lane and can potentially be explained by a homolgous 
recombination event due to the high sequence identity between the repeating GBP units. The appearance of the second band in the LamB-5RGBP induced culture may indicate a heterogeneous population or a degradation product of the LamB-5rGBP protein. Potential heterogeneity is further compounded due to the fact that $\mathrm{pBBAD} 18 \mathrm{~K}$ is not a tunable expression vector.

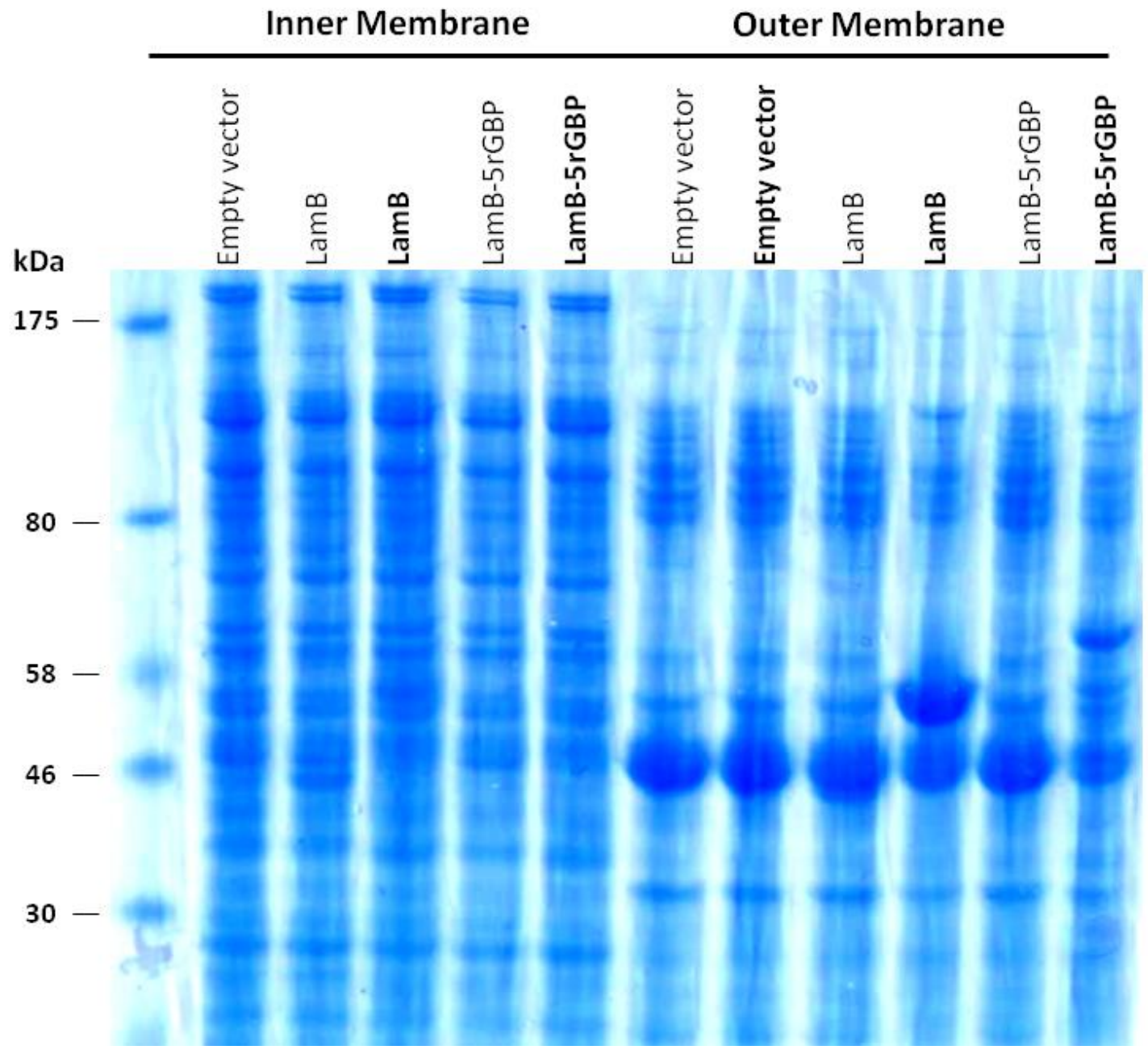

Figure 2.5. Membrane fractions subjected to SDS PAGE. Bold type indicates induction with 0.05\% L-arabinose at mid-log phase. Expressed LamB and LamB-5rGBP fusion proteins were found in the outer membrane.

To determine if the expression of LamB-5rGBP on the outer surface of the cell also increased adsorption to gold surfaces, induced cultures were incubated overnight with freshly cleaned gold wafers. The LamB-5rGBP fusion protein was not only transported to the outer membrane, but inserted in a manner that led to increased affinity for gold surfaces (Figure 2.6). 
Few cells bound to gold in strains expressing either the empty vector control or LamB. Bound cells were stained using a LIVE/DEAD kit and adsorption to the gold wafers was visualized using an inverted fluorescence microscope. Toxicity did not seem to be an issue for cells on gold as very few stained red, which would have indicated a compromised membrane. A total of six images were taken for each electrode, and representative images were chosen.

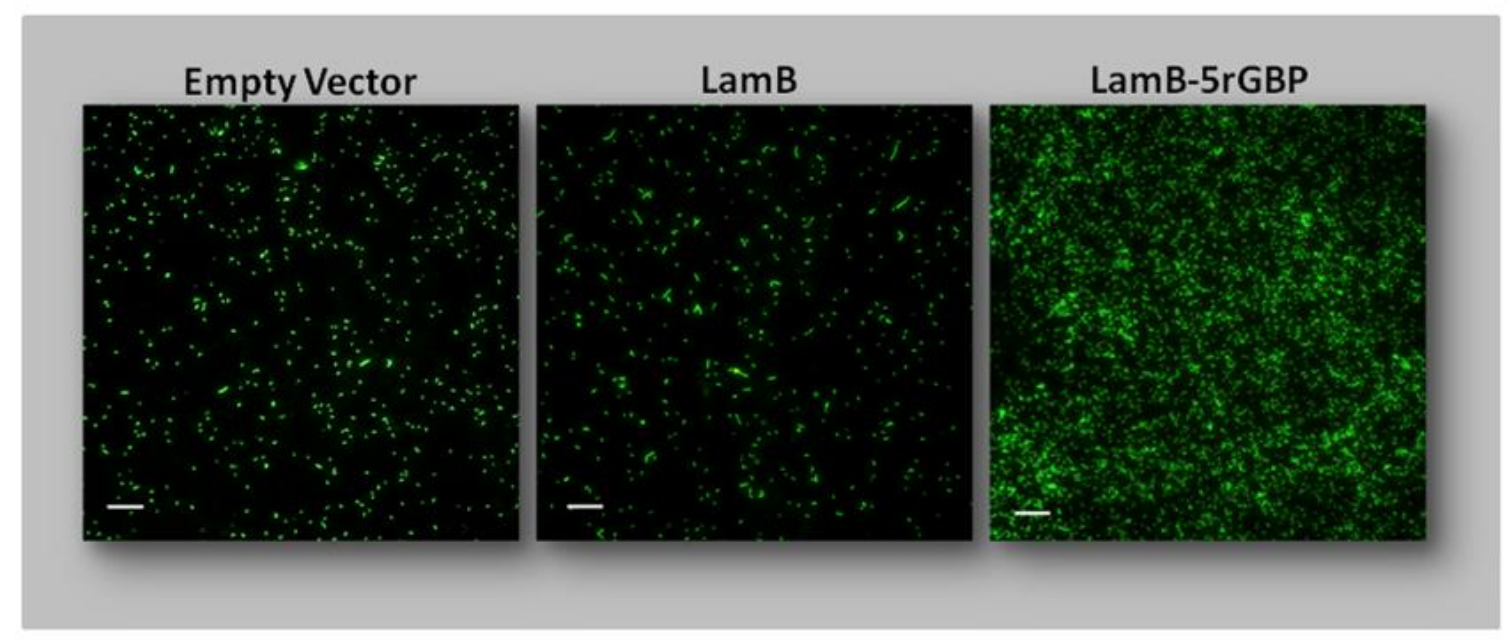

Figure 2.6. Adhesion to gold(111) plated silicon wafers. Gold wafers were added to stationary phase cultures and incubated at room temperature overnight. Bound cells were stained using the LIVE/DEAD BacLight ${ }^{\mathrm{TM}}$ Kit and were visualized using an Olympus IX70 inverted fluorescent microscope. Six images were taken of each wafer and representative images are shown. Scale bar is $20 \mu \mathrm{m}$.

Expression of these non-native proteins did not greatly affect growth rate (Figure 2.7), but did have adverse effects on other physiological aspects of $S$. oneidensis. The first effect noticed was a characteristic color change seen in cell pellets as is shown in the resuspended samples used for membrane fractionation (Figure 2.8C). A slight color change was seen in the strain expressing LamB, while the LamB-5rGBP strain exhibited the greatest shift and was orange in hue. The outer membrane of $S$. oneidensis contains multiple heme-containing $c$-type cytochromes that are important in extracellular respiration, and it is these proteins that give $S$. oneidensis its characteristic pink color. Therefore, it was reasoned that this loss of color in strains expressing either LamB or LamB-5rGBP could be due to the displacement of outer membrane cytochromes. 
As a dissimilatory metal reducer, $S$. oneidensis requires a system to facilitate the transfer of electrons from the cytoplasmic membrane to insoluble acceptors during extracellular respiration. The Mtr pathway, which includes the $o m c A-m \operatorname{tr} C A B$ gene cluster and its paralogs along with the tetra-heme cytoplasmic membrane protein CymA, provides this transport machinery (Coursolle and Gralnick, 2010). Electrons from reduced quinones are transferred to the inner membrane bound CymA (Schwalb et al., 2003; Shi et al., 2007) which then transfers them to the periplasmic deca-heme protein MtrA (Pitts et al., 2003). MtrA then facilitates the transfer of electrons to the surface-exposed deca-heme proteins MtrC and OmcA (Hartshorne et al 2009). The interaction between MtrC and MtrA is thought to be facilitated by the non-heme containing outer membrane porin MtrB (Hartshorne et a., 2009; Myers and Myers, 2002).

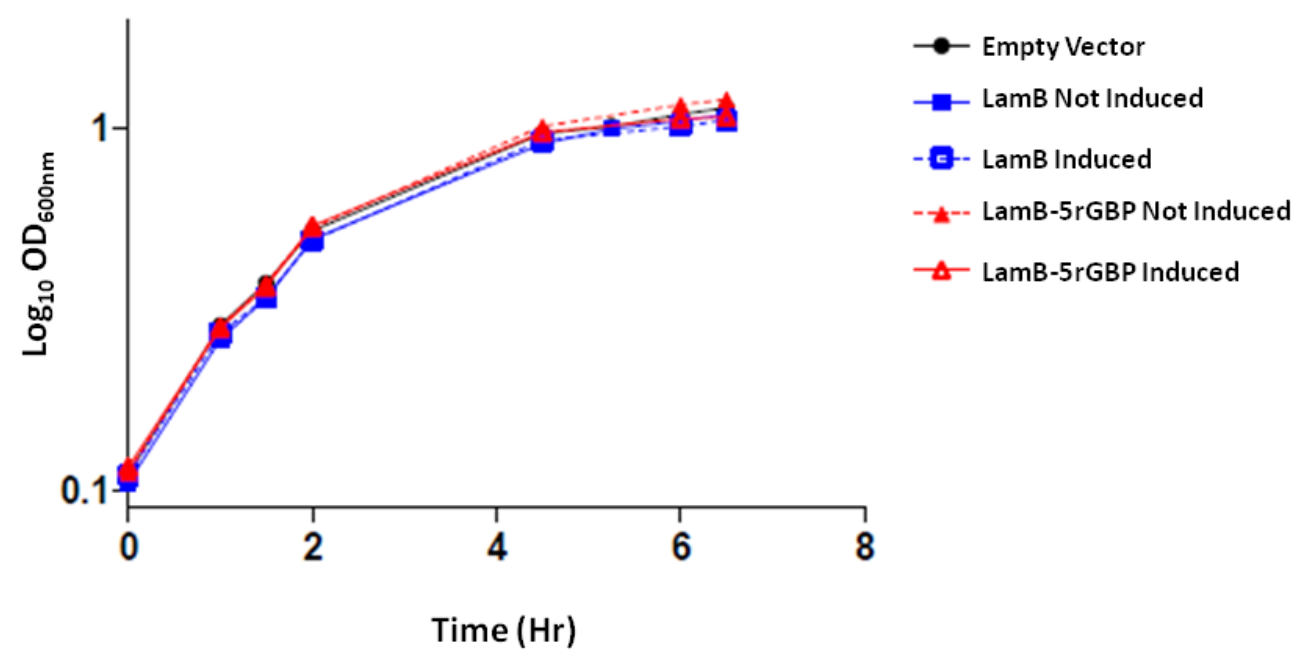

Figure 2.7. Aerobic growth in LB of the empty vector control, and strains engineered to express LamB and LamB-5rGBP. Filled and open shapes represent un-induced and induced cultures, respectively. Data shown is the average of cultures grown in triplicate.

The loss in pink color for cultures expressing either LamB or LamB-5rGBP led us to hypohesize that these proteins could potentially be displacing native Shewanella outer membrane components. To visualize potential cytochrome loss, sonicated whole-cell extracts were separated on $4-12 \%$ bis-tris gels under non-denaturing conditions and then heme stained. Results are outlined for the strain expressing LamB-5rGBP in Figure 2.8A and reveal that the amount of staining, which is proportional to protein levels, decreased for outer membrane cytochromes $\mathrm{MtrC}$ and OmcA in the induced culture. Interestingly, MtrA levels also appeared to decrease upon induction. 
Previous work by the Tien lab has shown that MtrA co-purifies with MtrC and MtrB as a complex, and fractionates with the outer membrane in sucrose density gradients (Ross et al., 2007). Recent research concerning this complex has also found that while the MtrAB complex forms in the absence of MtrC, the MtrBC complex cannot form in the absence of MtrA.

Furthermore, MtrA was only able to associate with the outer membrane in the presence of MtrB (Hartshorne et al., 2009). Therefore, the reduction in MtrA levels could potentially be explained by the displacement of MtrB from the outer membrane by either LamB or LamB-5rGBP. To test this, Western blots were performed for the membrane fractions using antibodies against MtrB. As predicted, the concentration of outer membrane MtrB was diminished in both LamB and LamB5rGBP expressing strains (Figure 2.8B).

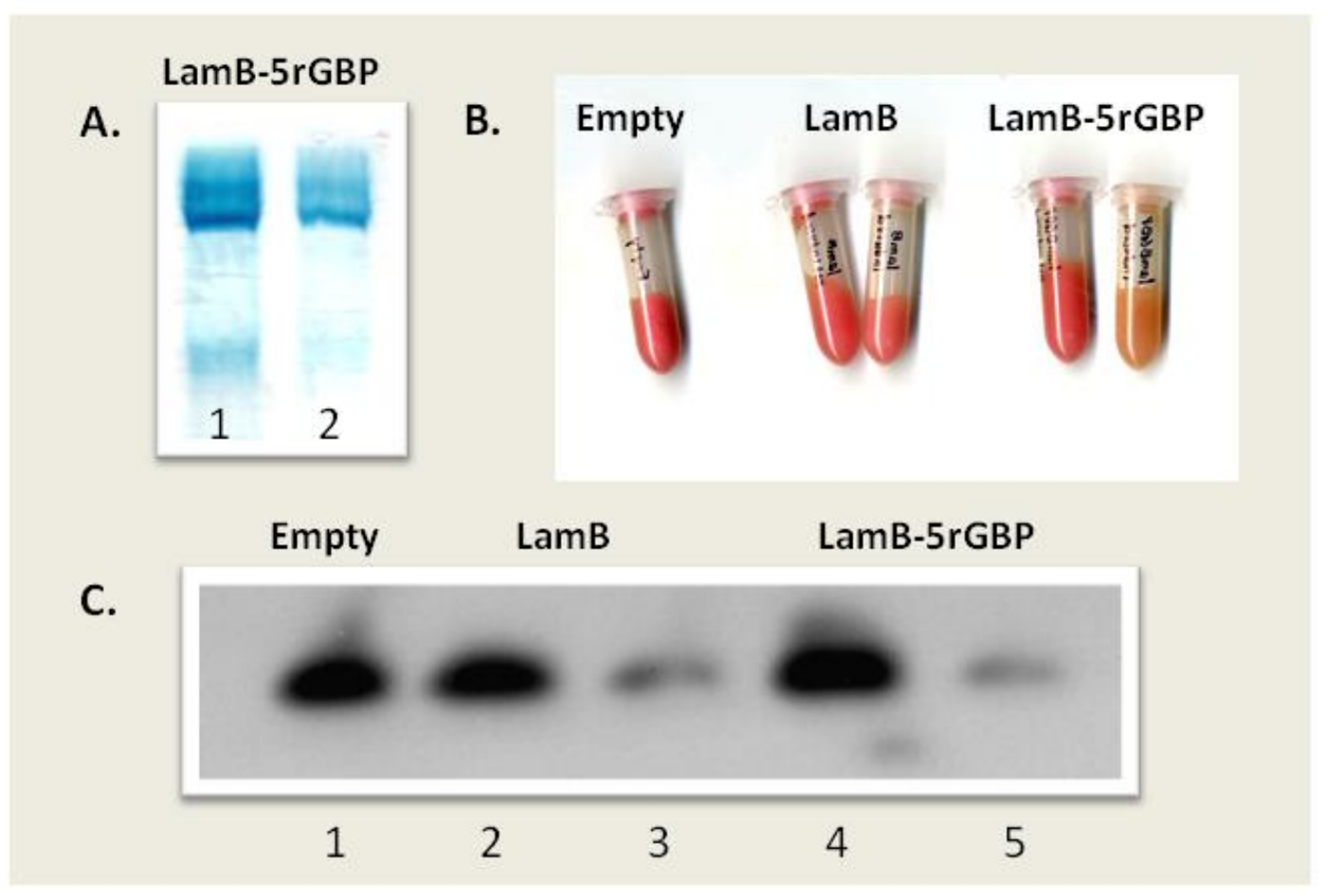

Figure 2.8. Expression of LamB and LamB-5rGBP leads to decreased levels of outer membrane proteins. A) Representative heme stain of outer membrane fractions from LamB-5rGBP cultures. Lane 1 is un-induced and lane 2 is induced sample. B) Color changes associated with LamB and LamB-5rGBP expression. Samples on the right in each grouping represent induced cultures. C) Western blot utilizing an antibody against MtrB. Lanes 15 correspond to Empty vector, LamB un-induced, LamB induced, LamB-5rGBP un-induced, and LamB-5rGBP induced, respectively. 
Since these outer membrane proteins are required for the transfer of electrons produced during metabolism to certain electron acceptors under anaerobic conditions, we next tested the ability of these strains to reduce both soluble and insoluble iron compounds. As can be seen in Figure 2.9, expression of both LamB and LamB-5rGBP impaired the rate at which these strains could reduce iron oxide. Effects in the reduction rate of iron citrate were not significant. It should also be noted here that the Mtr pathway is required for the reduction of electrodes in bioelectrochemical cells (Coursolle et al., 2010). Further data regarding the effects of the reduced levels of these proteins can be found in Chapter 3.

A
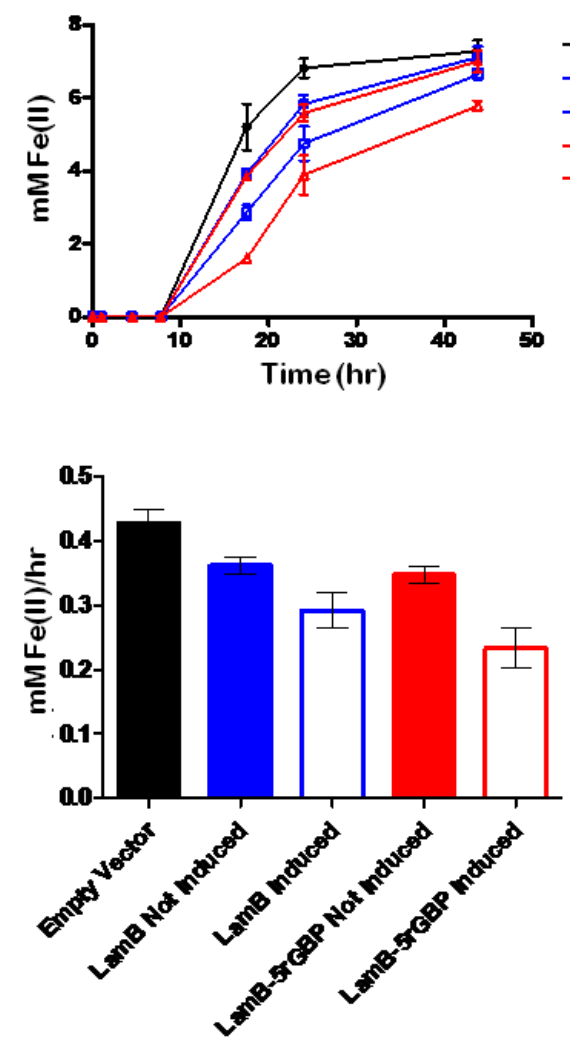

B
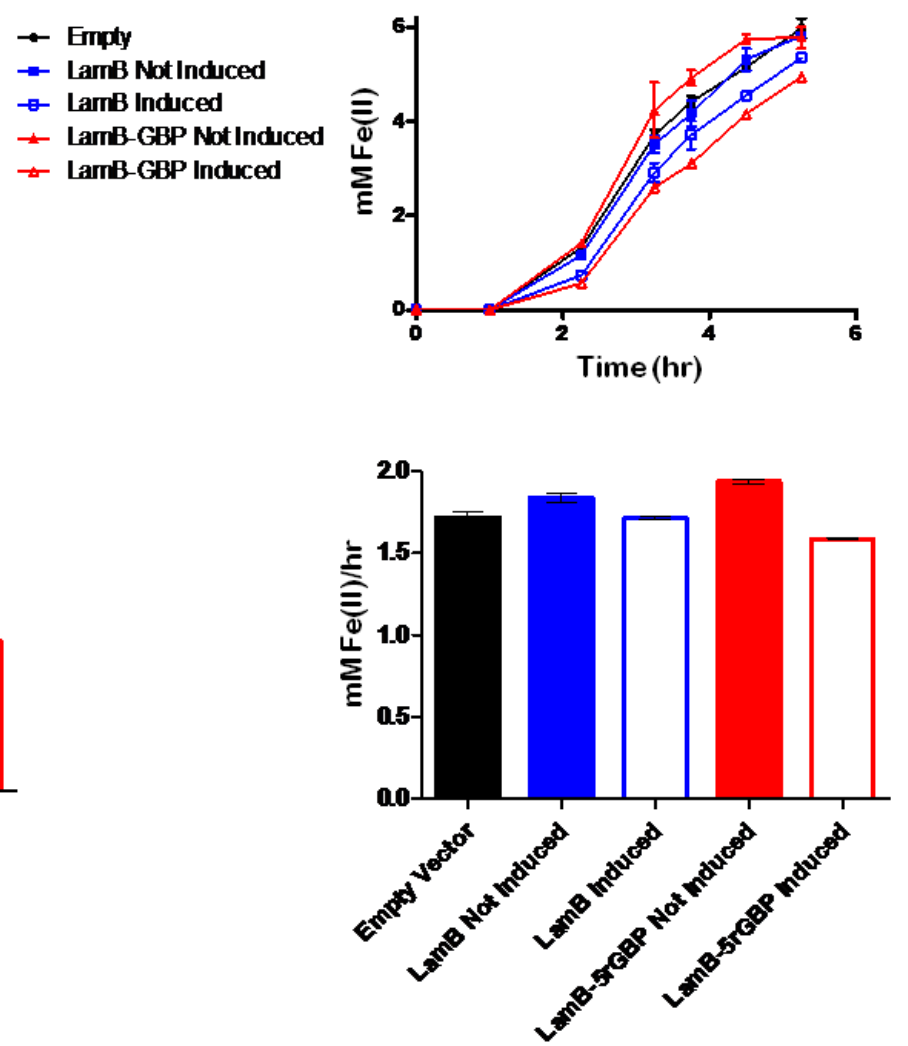

Figure 2.9. Effects of LamB and LamB-5rGBP on the reduction of both insoluble and soluble iron. A) (Above) Time course assay measuring the formation of $\mathrm{Fe}(\mathrm{II})$ during the reduction of insoluble iron oxide. (Below) Reduction rates of iron oxide. B) (Above) Time course assay measuring the formation of $\mathrm{Fe}$ (II) during the reduction of soluble iron citrate. (Below) Rates for the reduction of iron citrate. Rates were measured in the linear range of iron reduction assays. Assays were performed in triplicate and error bars represent the standard error. 


\section{Conclusion}

Previous research has indicated that Shewanella species are resistant to colonizing gold surfaces due either to an inability to bind and interact with the surface or due to some innate toxic property of gold itself. Upon further inspection, these results are not completely surprising since the gold surface differs greatly at the molecular level compared to the natural surfaces Shewanella encounters. One can assume that no strain has evolved to interact with such surfaces. By engineering a strain of $S$. oneidensis to display a modified $E$. coli outer membrane porin with an integrated gold-binding polypeptide (GBP-1), we were able to direct adsorption to gold(111). This is novel since prior methods to increase interaction of Shewanella and gold in reactor systems have had to rely on methods that modify the gold surface (this detail is covered in depth in Chapter 3). As to the toxicity of the gold surface, our results seem to indicate that this prediction is false since images of cells on gold mainly stained "live." Lastly, although expression of the heterologous protein LamB-5rGBP increased affinity of cells for gold surfaces, its expression also resulted in displacement of other outer membrane proteins; namely, the outer membrane decaheme $c$-type cytochromes and MtrB. This outcome could potentially lead to problems in further aspects of this research since these components are required for the reduction of electrodes. 


\section{Chapter 3}

\section{Electrochemical Analysis of Shewanella Strains Engineered to Express a Gold-Binding Polypeptide on Gold Electrodes}

\section{Introduction}

Shewanella oneidensis is a facultative anaerobe perhaps best known for its vast respiratory diversity. Under anoxic conditions, this organism can respire more than 20 different organic and inorganic compounds making it amenable to changing environments (reviewed in Hau and Gralnick, 2007; Nealson and Scott, 2006). As an added feat, many of these substrates are insoluble at or near neutral $\mathrm{pH}$ ranges, meaning that Shewanella require a specialized system to transfer electrons produced during carbon oxidation to extracellular substrates in a process termed dissimilatory metal reduction or extracellular respiration (Gralnick and Newman, 2007; Lovley, 2002). This respiratory diversity is not only important in biogeochemical cycling and bioremediation, but has also garnered interest for utilizing Shewanella in biotechnological applications.

The processes of respiration generally occur within the inner membrane, so extracellular respiration requires a specialized system. For the reduction of insoluble metals and electrodes, Shewanella utilizes the Mtr pathway, which includes the omcA-mtrCAB gene cluster, its paralogs, and the tetra-heme cytoplasmic membrane protein CymA (Coursolle and Gralnick, 2010). Current models for this pathway propose that electrons produced during carbon oxidation are transported from the menaquinone pool to the tetra-heme protein CymA, which acts as the terminal cytoplasmic membrane electron acceptor (Schwalb et al., 2003; Shi et al., 2007). These electrons are then passed to the periplasmic MtrA and finally to the terminal deca-heme cytochromes MtrC and OmcA located in the outer membrane (Hartshorne et al., 2009; Pitts et al., 2003). The interaction between MtrA and MtrC is facilitated by the integral outer membrane protein MtrB (Hartshorne et al., 2009; Myers and Myers, 2002).

Bioreactors provide a unique tool to simulate the natural transfer of electrons from carbon oxidation to insoluble electron acceptors and enable these processes to be analyzed quantitatively. 
In our systems, Shewanella oxidizes lactate, which acts as an electron donor and utilizes an anodic electrode as the final acceptor. It should be noted that the bioreactors utilized in this study differ slightly from microbial fuel cells (MFCs). Contrary to MFCs, our reactor set-up uses a single chamber and the classical three-electrode system, which allows a user-defined potential to be set at the working electrode via a reference electrode and potentiostat (Figure 3.1). By linking these processes to bio-electrochemical reactor circuitry, one can monitor the process of extracellular respiration.

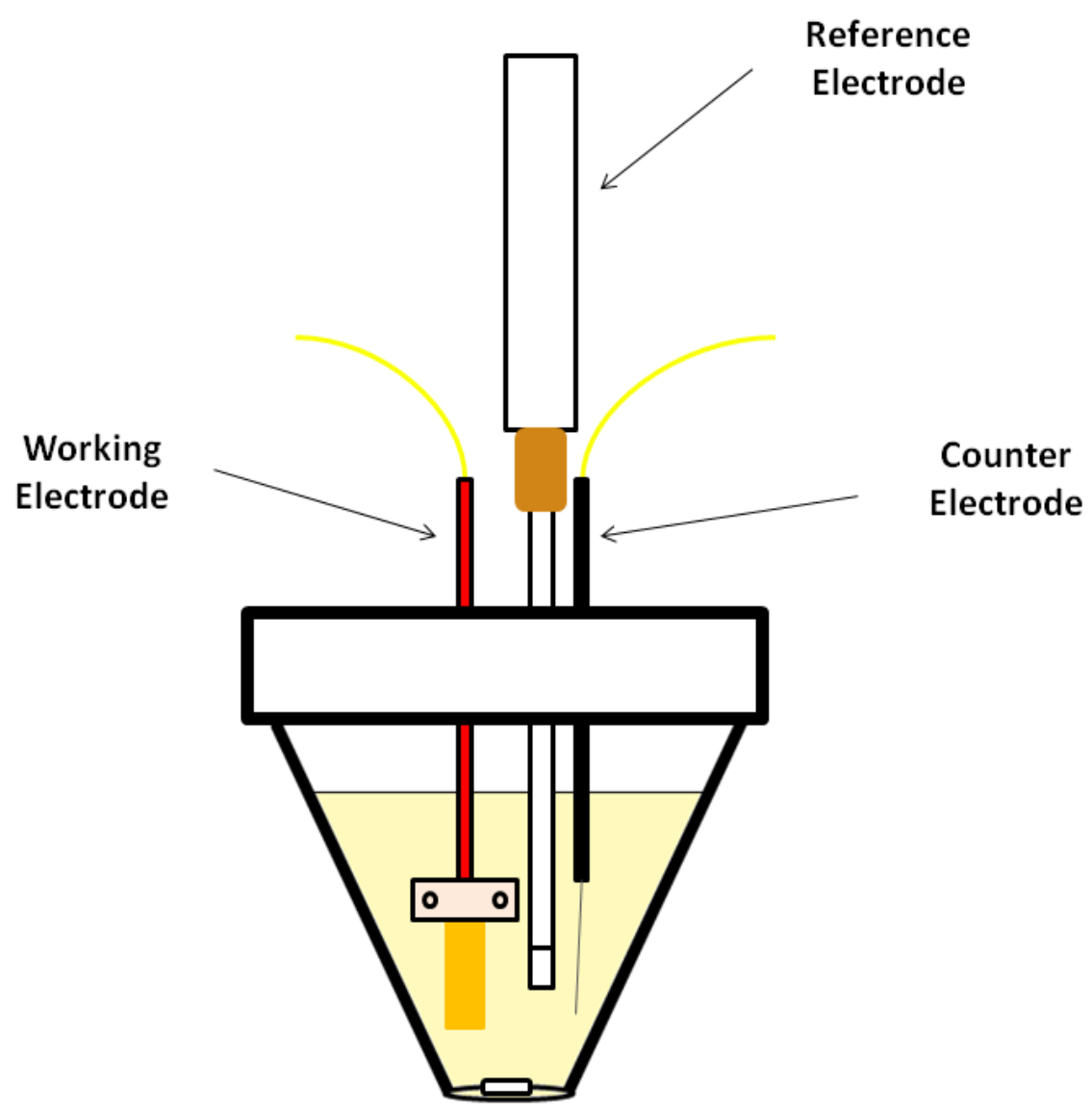

Figure 3.1. Schematic displaying the three-electrode bioreactors utilized in this study. A constant potential of 0.24V versus SHE (Standard Hydrogen Electrode) is set at the working electrode using a multichannel potentiostat. Current can then be measured between the working and counter electrodes.

This study sought to utilize the extracellular respiratory mechanisms of a strain of $S$. oneidensis engineered to bind gold surfaces in bioreactors with a gold electrode serving as the 
anode - a feat previously only made possible by altering the gold surface. Gold exhibits multiple characteristics that make it an attractive material for anode construction. Although expensive, gold electrodes are highly conductive, resistant to oxidation, and can be manufactured to display highly-defined and well-studied surface topologies. Perhaps most important is the fact that gold electrodes exhibit high signal-to-noise ratios during data collection compared to the more common electrode material, graphite.

Despite the aforementioned qualities, previous research has shown that bare gold is a poor choice as an anode material when it comes to reactors utilizing Shewanella (Crittenden et al., 2006; Sun et al., 2010). Multiple theories as to why Shewanella fails to interact with bare gold electrodes have been posed and include: toxicity of the surface, lack of functional groups that mediate microbe-electrode interactions, coupling issues due to a block in electron transport from the bacterium to the surface, inability to bind, and instability of mediators in redox reactions with gold. To overcome these issues and encourage the coupling of electron transfer mechanisms to gold surfaces, previous studies have relied on varying the electrode surface itself. Formation of an acid-terminated self-assembled monolayer (SAM) on the gold surface allowed current production in MFCs; whereas, bare gold exhibited only a capacitive discharge (Crittenden et al., 2006). Sputtering of a gold layer atop graphite electrodes also increased current production when compared to that of bare gold (Sun et al., 2010). The goal of our study was to engineer a strain of Shewanella with the ability to bind gold surfaces thereby creating a self-generating answer to the problem of current generation on bare gold without relying on modification of the surface itself.

Many studies have alluded to the potential toxicity of the gold surface and the possibility of strong adsorption leading to denaturing of outer membrane proteins, yet data from this and other studies seems to indicate that is not the case for Shewanella (Crittenden et al., 2006; Sun et al., 2010). The vast majority of S. oneidensis cells imaged on gold surfaces in Chapter 2 of this study stained "live" indicating that membranes had not been compromised. Also, scanning tunneling microscopy and tunneling spectroscopy studies have characterized the electron tunneling properties of the outer membrane cytochromes MtrC and OmcA by immobilizing them on gold(111) surfaces (Wigginton et al., 2007a and b). This may also negate the idea of a block in electron transport between the outer membrane cytochromes and gold - the caveat being that these experiments were conducted by specifically immobilizing the proteins to allow electron tunneling. It is also interesting to note that not all electrogenic bacteria exhibit this inability to 
couple with bare gold electrodes as previously cited. Studies using Geobacter sulfurreducens, another organism capable of extracellular respiration, produced current both in microbial fuel cells and in bioreactors using bare gold anodes (Richter et al., 2008; Liu et al., 2010).

Other explanations for the lack of current generation in reactors utilizing Shewanella and gold anodes focus on interactions at the microbe-electrode and mediator-electrode interfaces. Gold electrodes differ greatly, both chemically and physically, at the molecular level compared to substrates that Shewanella species encounter naturally. Some have posed that functional groups similar to humic substances found in soil and sediment environments may be important in mediating electron transfer to electrodes (Crittenden et al., 2006; Lovley et al., 1996; Richter et al., 2008). Humates are a heterogeneous organic material abundant in soils and sediments that can be used as an electron acceptor and potential mediator in anoxic environments (Lovley et al., 1996). Graphite electrodes contain many of the functional groups present in humic substances while gold does not, and this may account for the difference in electron transfer seen between gold and graphite electrodes. Shewanella also has been shown to secrete redox-active flavins which promote the reduction of metals and electrodes (Marsili et al, 2008; von Canstein et al, 2008). Prior to this study, it was unknown if flavins remained chemically stable during reactions with gold. This study revealed that flavins can promote the reduction of gold surfaces, and that this reaction is reversible.

The failure to electronically couple with bare gold electrodes could potentially be due to inefficient binding and hence biofilm formation on the gold surface. SEM images from Sun et al revealed that layers of $S$. oneidensis were observed on gold-sputtered carbon anodes; whereas, bare gold contained only a few cells sporadically located along the surface (2010). This contradicts data found in the Crittenden et al study which states that bare gold surfaces contained four times more cells than SAM-modified gold (2006). It must be noted here that these studies not only utilized different strains, S. oneidensis and S. putrefaciens, respectively, but also different incubation conditions with gold. SEM images were taken from MFC electrodes utilized in data collection for the Sun study, while the Crittenden study incubated mid-logarithmic cells with gold surfaces for two hours.

By engineering a strain of $S$. oneidensis able to bind gold surfaces, we hoped to overcome the inability of Shewanella to couple with gold electrodes without relying on modifying the 
electrode surface. This study was also the first to look at the role of binding in current generation on bare gold surfaces. If the lack of current generation is resultant only from Shewanella's inability to form biofilms on gold, then reactor analysis utilizing our engineered gold-binding strain should result in overall increased current production. Due to its importance as a mediator in electron transfer in bioreactor systems, the stability of riboflavin on gold was also examined in this study.

\section{Materials and Methods}

\section{Bacterial Culturing and Growth}

Strains and plasmids used in this study are outlined in Table 3.1. All overnight cultures were inoculated from single colonies taken from freshly streaked $-80^{\circ} \mathrm{C}$ stocks on $\mathrm{LB}$ plates containing $50 \mu \mathrm{g} / \mathrm{ml}$ kanamyacin. These cultures were grown for 16 hours in Luria-Bertania (LB) medium supplemented with $50 \mu \mathrm{g} / \mathrm{ml}$ kanamycin $(\mathrm{Km})$ to support the maintenance of plasmids. Unless specified otherwise, cultures were shaken continuously at $250 \mathrm{rpm}$ and incubated at $30^{\circ} \mathrm{C}$.

\begin{tabular}{lll}
\hline Strain or Plasmid & Genotype/Characteristics & Reference/Source \\
\hline $\begin{array}{l}\text { S. oneidensis strain } \\
\text { MR-1 }\end{array}$ & Isolated from L. Oneida, NY & $\begin{array}{l}\text { Venkateswaran et al, } \\
1999\end{array}$ \\
JG1301 & $\begin{array}{l}\text { S. oneidensis MR-1: empty } \\
\text { pBBAD18K vector }\end{array}$ & This Study \\
& $\begin{array}{l}\text { S. } \text { oneidensis MR-1: } \operatorname{lamB} \\
\text { JG1302 }\end{array}$ & $\begin{array}{l}\text { This Study } \\
\text { JG1303 }\end{array}$ \\
\end{tabular}

\begin{tabular}{lll}
\hline \hline pBBAD18K & 7.6 kb broad host range cloning vector; & Sukchawalit et al., \\
L-arabinose induced $; \mathrm{Km}^{\mathrm{R}}$ & 1999
\end{tabular}

plamB

pBBAD18K containing lamB (cloned This Study from E. coli $\mathrm{K} 12$ strain MG1655); modified at codons $155-157$ to introduce PstI and XhoI restriction sites 
Table 3.1. Strains and plasmids utilized in this study.

\section{Bioreactor Construction}

Bio-electrochemical reactors utilized in this study consisted of a $25 \mathrm{~mL}$ glass cone (Bioanalytical Systems, West Lafayette, IN) sealed by a Teflon top modified to hold electrodes and a gas line, which maintained anaerobic conditions by flushing the reactor with humidified nitrogen (Figure 3.1). These reactors were set up using the classical three-electrode system. A silicon wafer electroplated with a 1000 A thick layer of gold(111) cut to 1" x 1/4" served as the working electrode and was connected to a platinum wire using a plastic clamp and Teflon bolts (Small Parts, Inc., Miramar, FL). Prior to cleaning, gold electrodes were stored in acetone overnight, followed by ethanol for at least one hour to remove any organic material. Gold electrodes were then further cleaned in a 3:1 (conc. sulfuric acid: $30 \%$ hydrogen peroxide) piranha solution for 20 minutes followed by three washes in filtered and autoclaved $\mathrm{ddH}_{2} \mathrm{O}$. These electrodes were dried under filtered nitrogen and used immediately. A platinum wire was also used as the counter electrode which was cleaned for 24 hours in $1 \mathrm{~N} \mathrm{HCl}$ prior to use. The reference consisted of a glass body saturated calomel electrode (Fischer Scientific, Pittsburgh, PA) connected to the system via a glass capillary tube filled with $1 \%$ agarose in $0.1 \mathrm{M}$ sodium sulfate and capped with a vycor frit. Reactors were autoclaved following construction and used immediately. Electrochemical data was monitored using a 16-channel VMP® potentiostat (Bio-Logic, Claix, France).

\section{Current Analysis in Reactors Poised During Growth}

Sealed anaerobic Balch tubes containing $10 \mathrm{~mL}$ of LB supplemented with $100 \mathrm{mM}$ HEPES buffer, $30 \mathrm{mM}$ lactate, $40 \mathrm{mM}$ fumarate, and $50 \mu \mathrm{g} / \mathrm{mL} \mathrm{Km}$ were inoculated to an $\mathrm{OD}_{600 \mathrm{~nm}} \sim 0.05$ from an aerobic LB overnight. Cultures were induced with $0.05 \%$ L-arabinose at an $\mathrm{OD}_{600 \mathrm{~nm}} \sim$ 0.5 . After reaching stationary phase, cultures were transferred to anaerobic reactors via a nitrogen-flushed syringe and were stirred continuously and kept at $30^{\circ} \mathrm{C}$. The working electrode was then poised at $0.24 \mathrm{~V}$ versus SHE and $30 \mathrm{mM}$ lactate and $1 \mu \mathrm{M}$ riboflavin were added to the 
reactor. Reactors were monitored by chronoamperometry and, due to low current levels after 24 hours, were spiked with $10 \mu \mathrm{M}$ riboflavin.

\section{Current Analysis in Reactors Not Poised During Growth}

Aerobic LB overnight cultures were used to inoculate anaerobic Balch tubes containing $10 \mathrm{~mL}$ of LB medium buffered with 100mM HEPES and supplemented with $30 \mathrm{mM}$ lactate, $40 \mathrm{mM}$ fumarate, $0.5 \mu \mathrm{M}$ riboflavin, and $50 \mu \mathrm{g} / \mathrm{mL} \mathrm{Km}$ to an $\mathrm{OD}_{600 \mathrm{~nm}} \sim 0.05$. When cells reached midlogarithmic phase, $\mathrm{OD}_{600 \mathrm{~nm}}=0.5$, cultures were induced with $0.05 \% \mathrm{~L}$-arabinose and transferred to anaerobic reactors using a syringe flushed with nitrogen. Reactors were kept at $30^{\circ} \mathrm{C}$ using a water bath and stirred continuously for 16 hours. To remove planktonic cells after this incubation period, three media replacements were conducted with LB containing $100 \mathrm{mM}$ HEPES, $30 \mathrm{mM}$ lactate, and $1 \mu \mathrm{M}$ riboflavin. The working/gold electrode was then poised at 0.24 versus SHE and current production was monitored by chronoamperometry using a multichannel potentiostat.

Reactors were run for approximately 24 hours after which electrodes were removed and processed for imaging.

\section{Imaging of Electrodes}

Electrodes were removed from reactors and immediately rinsed once in SBM. This minimal wash procedure was chosen in an attempt to maintain a surface as similar as possible to the one analyzed during electrochemical measurements. Bound cells were stained using the LIVE/DEAD BacLight ${ }^{\mathrm{TM}}$ Bacterial Viability Kit (Kit L7012) from Invitrogen according to the manufacturer's instructions. Fluorescence was then visualized using an Olympus IX70 inverted microscope and analyzed utilizing the SPOT ${ }^{\mathrm{TM}}$ Basic software. Six images were taken of each surface, and representative images were chosen.

\section{Riboflavin Reduction Assay}

Aerobic LB overnight cultures were used to inoculate fresh $2 \mathrm{~mL} \mathrm{LB}$ cultures to an $\mathrm{OD}_{600 \mathrm{~nm}}$ 0.08 and these cultures were induced with $0.05 \%$ L-arabinose at an $\mathrm{OD}_{600 \mathrm{~nm}} \sim 0.5$. After reaching stationary phase, cells were spun down and resuspended to an $\mathrm{OD}_{600 \mathrm{~nm}}=1$ in $\mathrm{SBM}$ containing $0.05 \%$ arabinose. In an anaerobic chamber, $20 \mu \mathrm{L}$ of the resuspended samples were added to black 96-well plates containing $280 \mu \mathrm{L}$ of SBM supplemented with $20 \mathrm{mM}$ lactate, $133 \mu \mathrm{M}$ riboflavin, vitamins, minerals, and $0.05 \%$ L-arabinose. Fluorescence was read (excitation $=440$ 
$\mathrm{nm}$, emission $=525 \mathrm{~nm}$ ) for 24 hours at 30 -second intervals. Experiments were performed in triplicate to obtain accurate reduction rates.

\section{Reduction of $\mathrm{Au}(111)$ Electrodes By Riboflavin}

Reactors were constructed as described in previous paragraphs. Base-line cyclic voltammetry (CV) was conducted using blank anaerobic LB media with electrodes poised at $0.24 \mathrm{~V}$ versus SHE from -0.56 to $0.44 \mathrm{~V}$ at a scan rate of $1.0 \mathrm{mV} / \mathrm{s}$. To determine the effect of $\mathrm{LB}$ in data output, a second reactor was set up similarly containing blank SBM. LB (or SBM) media containing $100 \mu \mathrm{M}$ riboflavin was then added to reactors and CV analysis was performed again using the ranges and scan rate afore described.

\section{Results and Discussion}

Although gold is highly conductive, its use as an anode material in fuel cells produces little, if any, current with wild-type Shewanella. Previous attempts to increase current production and overcome the issues of colonization and coupling with gold have relied on modifying the surface, either through acid-terminated self-assembled monolayers or by sputtering gold atop carbon electrodes (Crittenden et al., 2006; Sun et al., 2010). By engineering a strain of Shewanella specific for adsorption on gold surfaces, this study represents the first effort at overcoming bio-incompatibility issues by reconfiguring the bacterium—not the surfaceresulting in a self-generating answer to the problem.

It was immediately realized that conventional methods for monitoring and analyzing biofilm growth would be unsuccessful when using gold electrode reactors even with the strain engineered to bind gold. After inoculation, typical reactors exhibit a capacitive discharge followed by an increase in current indicating cell attachment and biofilm formation. After a plateau in current, media replacements are performed to remove planktonic cells and electrochemical measurements based specifically on biofilm-associated cells are then collected. In reactors inoculated with either the empty vector control or the strain engineered to express LamB-5rGBP, chronoamperometry (CA) data was dominated by capacitive discharge and showed no following increase in current (Figure 3.2). The addition of $10 \mu \mathrm{M}$ riboflavin resulted in an immediate current spike, but no further elevation in current was observed. 
A

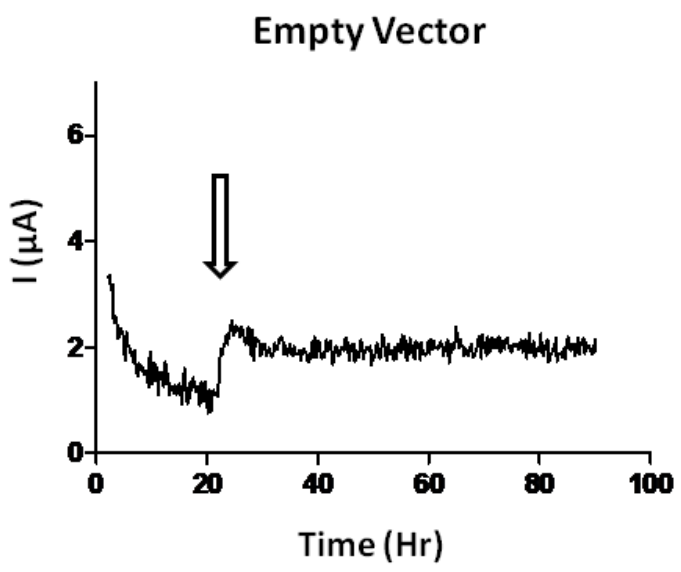

B

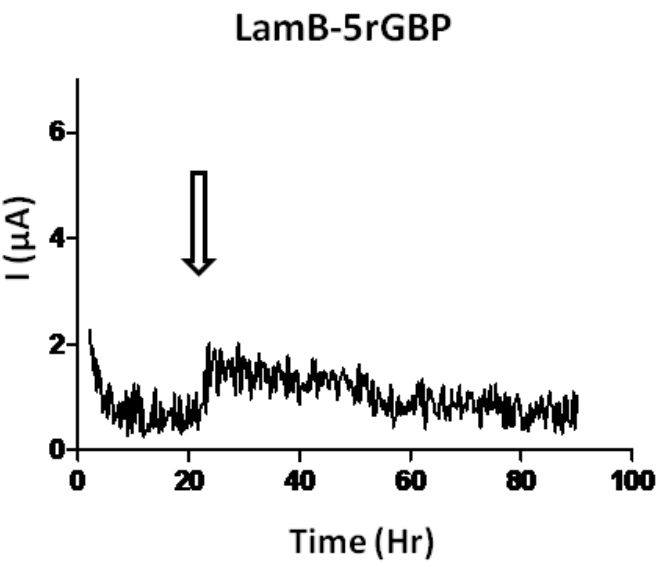

Figure 3.2. Chronoamperometry analysis prior to media replacement monitoring biofilm formation on gold.

Gold electrodes were poised at $0.24 \mathrm{~V}$ versus SHE. At the time of inoculation, $20 \mathrm{mM}$ lactate and $1 \mu \mathrm{M}$ riboflavin were added to the media. Arrows indicate the further addition of $10 \mu \mathrm{M}$ riboflavin in an attempt to increase current production. A) Current generated by the empty vector control and B) the induced LamB5rGBP strain.

Several possibilities were put forth to explain the lack of current in CA analysis following inoculation. We began by first looking at the effect LamB-5rGBP expression, and the resulting displacement of outer membrane proteins had on potential methods utilized by Shewanella to reduce extracellular substrates. Three main strategies have been posed to account for the transfer of electrons to insoluble acceptors - direct contact, small molecule shuttles or chelators, and conductive appendages (reviewed in Gralnick and Newman, 2007). The first indicates that direct contact between the solid surface and outer membrane cytochromes MtrC and OmcA can mediate electron transfer and substrate reduction. Research utilizing purified proteins suggests this mechanism likely plays a role (Firer-Sherwood et al., 2008). Small molecules such as chelators or shuttling mediators may also be involved. Shewanella secretes redox-active flavins, which have been shown to increase the rate of insoluble iron and electrode reduction (Marsili et al., 2008; von Canstein et al., 2008). Since observations indicate that Shewanella can reduce insoluble iron, both by direct contact and indirectly, it is likely that both direct contact and mediator strategies play a role in the reduction of extracellular electron acceptors (Lies et al., 2005). Lastly, electrically conductive pili or 'nanowires' have been observed by some groups and may also play a role (Gorby et al., 2006). 
As was outlined in Chapter 2, the expression of LamB-5rGBP led to the displacement of the outer membrane protein MtrB and cytochromes MtrA, MtrC, and OmcA (Fig. 2.8), which have been shown to be critical for the reduction of electrodes and flavins (Coursolle et al., 2010). This could potentially reduce the transfer of electrons mediated both by direct contact and via redox-active mediators. Since the Mtr pathway is essential for riboflavin reduction, we conducted an assay to measure the rate of riboflavin reduction by our engineered strains. Oxidized flavins are fluorescent whereas the reduced species are not; therefore, flavin reduction can be monitored using a spectrophotometer set up to measure fluorescence (Ghisla et al., 1974). Compared to the empty vector control, the rate of riboflavin reduction was reduced approximately 2 -fold for strains expressing either LamB or LamB-5rGBP (Fig. 3.2) and mirrored the results of insoluble iron reduction (Fig. 2.9). The decrease in reduction rates for un-induced cultures could potentially be due to basal expression by the $\mathrm{P}_{\mathrm{BAD}}$ promoter even in the absence of arabinose. Although overall rates were lessened in the LamB-5rGBP strain, riboflavin was still reduced. Hence, these results alone do not explain the lack of current production in reactors containing the strain engineered to adsorb onto gold.
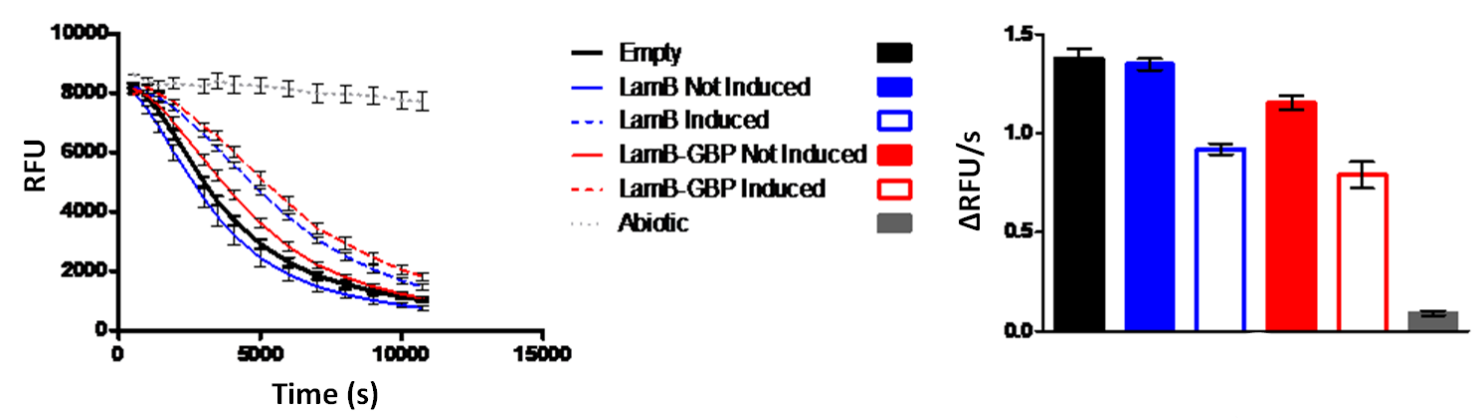

Figure 3.3. Rates of riboflavin reduction by strains engineered to express LamB or LamB-5rGBP as compared to the empty vector control. Reduction was monitored as the loss of fluorescence due to the reduction of riboflavin and reduction rates were calculated using the linear portions of the reduction assay curves. Error bars are representative of standard errors (SEM) for reduction rates calculated from three biological replicates analyzed in triplicate.

After being reduced by the cell, flavins diffuse through the media where they can come into contact with and reduce electrode surfaces. Previous research has briefly posed that the shuttling mechanism utilized by Shewanella, in contrast to the direct method of $G$. sulfurreducens, may not be conducive to reducing gold surfaces perhaps due to some chemical 
instability of flavins on gold (Richter et al., 2008). We tested this hypothesis by directly reducing gold electrodes with flavin enriched media. As indicated in the Materials and Methods section, abiotic reactors were set up containing $100 \mu \mathrm{M}$ riboflavin in either LB or SBM and cyclic voltammetry $(\mathrm{CV})$ was performed at a slow scan rate.

It is helpful to keep a few details in mind when analyzing the following CV data. During a CV trace or 'sweep', the potential at the working electrode is ramped linearly from a userdefined potential to an end potential and back again. Current, measured between the working and counter electrode, is plotted for each of these potentials. Positive current represents the oxidation of the mediator and reduction, or flow of electrons into the electrode; whereas, negative current represents the reverse- flow of electrons from the electrode which can then be used to reduce the mediator (Figure 3.4).
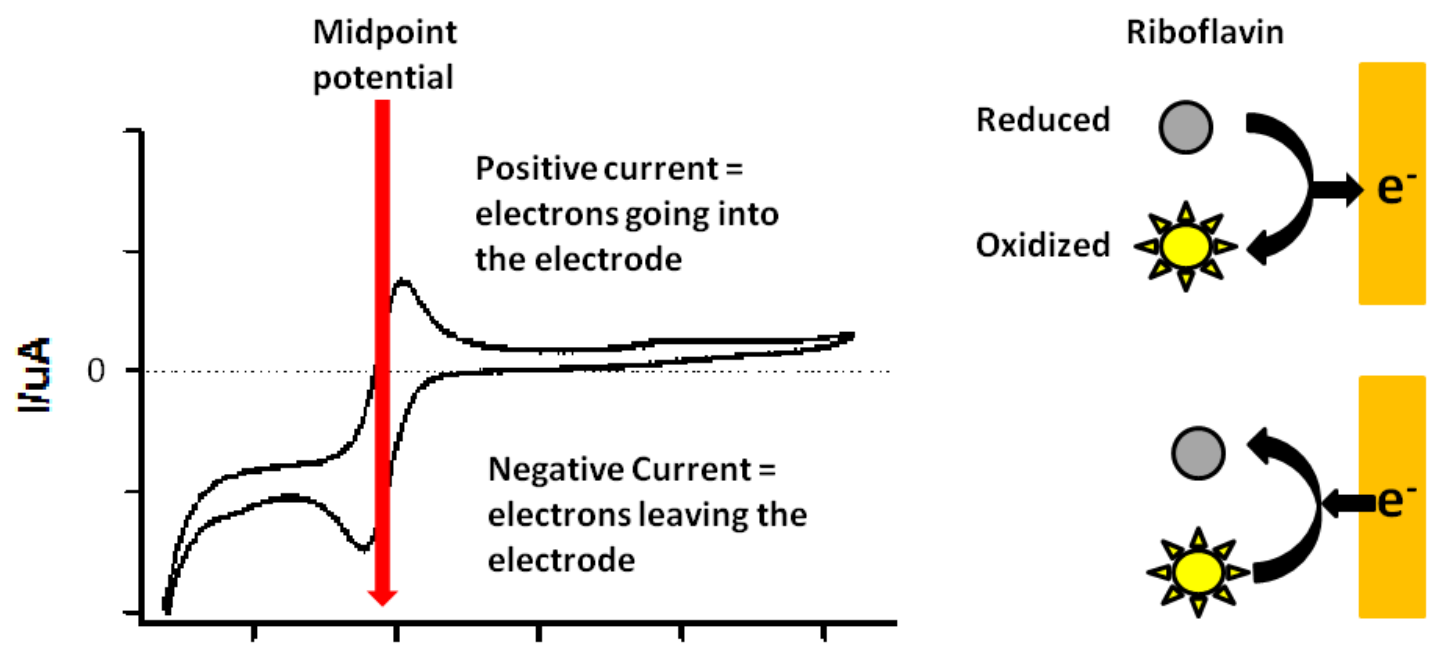

\section{Ewe/V}

Figure 3.4. Schematic outlining CV graphical features important for understanding data analysis. The midpoint potential occurs where the concentration of reduced and oxidized species are equal.

Two CV sweeps were performed, and the second is shown in Figure 3.5 for both LB and SBM. Since oxidized riboflavin is reduced below its midpoint potential, initial data points for the anodic sweep began at a negative current. This current increased, indicating reduction or flow of electrons into the electrode, as the working electrode ramped towards a more positive potential. A steep slope as the potential reached the reduction potential of riboflavin indicated that reduced 
riboflavin within the diffusion layer was quickly oxidized. Reduction of the electrode by oxidized flavins looked to be limited only by diffusion of the substrate and not by kinetics of the reaction since the peak was well-defined and quickly exhausted. The cathodic sweep also revealed a steep negative current with an inflection point at the midpoint potential of riboflavin; therefore, electrons from gold could effectively "re-reduce" riboflavin - i.e. the process was reversible. Data from $\mathrm{CV}$ analysis indicated that riboflavin remained stable in redox reactions with gold and was capable of reducing gold electrodes in bioreactor experiments.

A

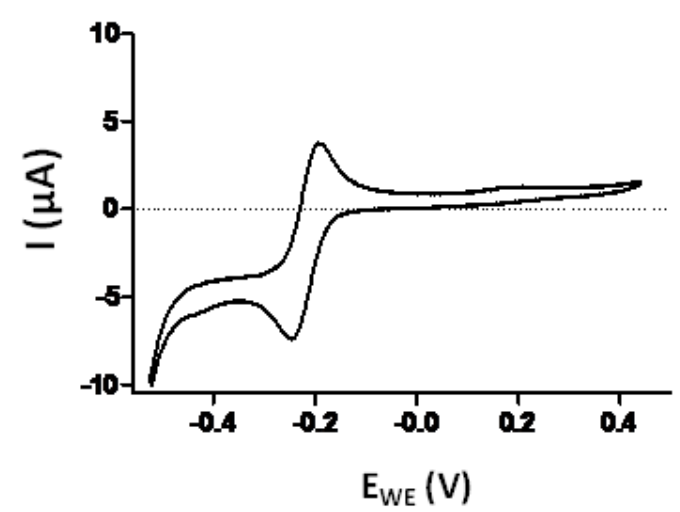

B
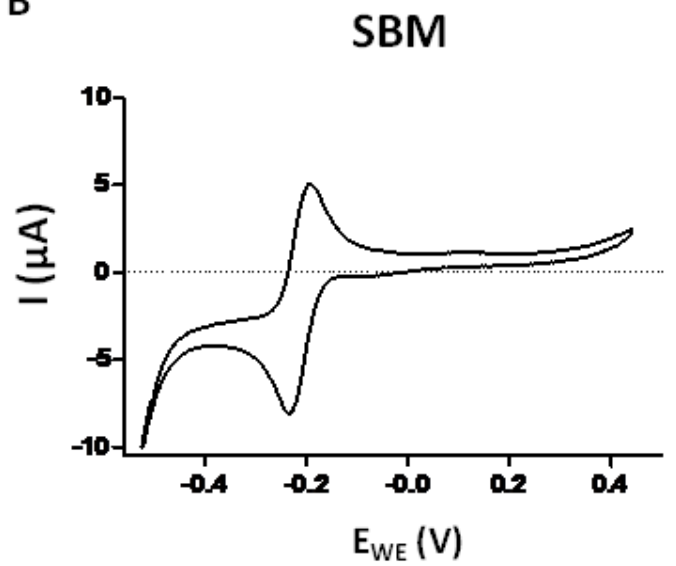

Figure 3.5. Cyclic voltammetry of the direct reduction of gold electrodes via $100 \mu \mathrm{M}$ riboflavin in A) LB and B) SBM. Current increased as the potential at the working electrode $(\mathrm{Ewe} / \mathrm{V})$ reached the reduction potential of riboflavin. The reverse scan indicates oxidized flavins could be reduced again by the electrode, i.e. the process is reversible.

Data, up to this point, indicated that each step involving the movement of electrons during extracellular respiration of gold electrodes worked when analyzed individually. The LamB-5rGBP strain could reduce riboflavin; riboflavin was able to reduce gold electrodes, yet when the gold-binding engineered strain of Shewanella was added to reactors with gold, very little current was produced. Poised gold surfaces exhibit a different surface charge compared to un-poised gold; therefore, poising at a positive potential leads to a positively-charged electrode surface. Gold-binding peptide (GBP-1) has an overall positive charge (+1); therefore, it is possible that poising the electrode at a positive potential repelled LamB-5rGBP and hence the biofilm. 
To overcome issues of binding to poised surfaces, bioreactors were constructed and allowed to form biofilms under un-poised conditions. Reactors were inoculated with midlogarithmic phase cultures, induced with arabinose, and then allowed to form films on un-poised gold surfaces overnight. After a 16-hour incubation period, three media replacements were performed to remove planktonic cells and CA analysis was initiated-i.e. electrodes were then poised. For the strain expressing LamB-5rGBP, initial capacitive discharge was followed by a sharp increase in current that peaked around $4.5 \mu \mathrm{A}$ after 8 hours and then fell to a steady baseline current slightly above $2 \mu \mathrm{A}$ for the remainder of the analysis (Figure 3.6A). The empty vector control strain quickly fell to background current levels immediately after being poised (Figure 3.6B). Following this analysis, electrodes were collected and processed for imaging.

A

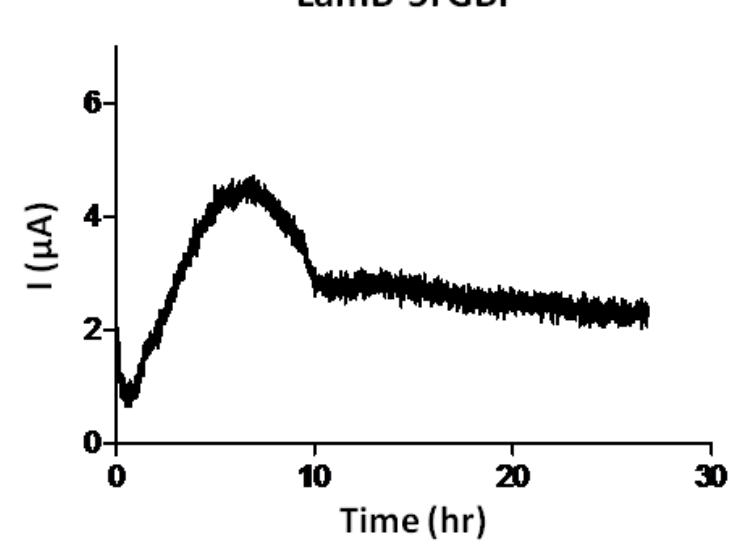

B

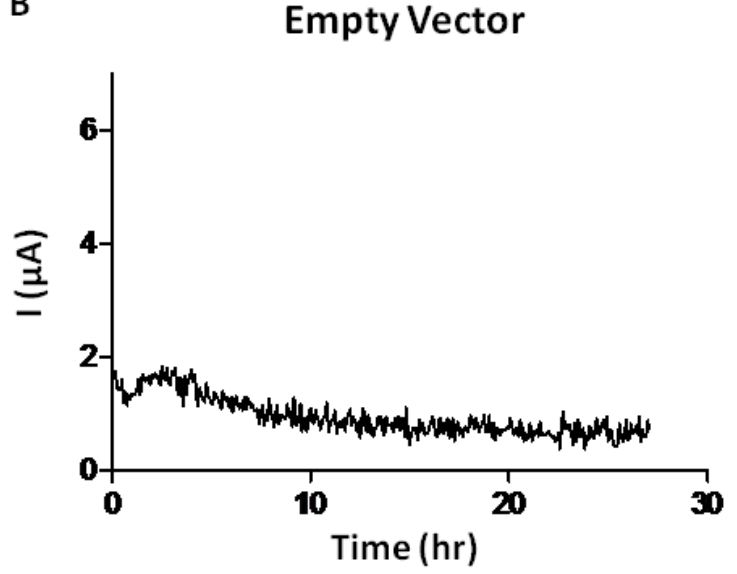

Figure 3.6. CA analysis of the gold-binding strain and the empty vector control after three media replacements to remove planktonic cells. A) Current in the strain expressing LamB-5rGBP increased after the discharge period, but peaked at approximately 8 hours to $\sim 4.5 \mu \mathrm{A}$ and fell to a baseline between 2 to $3 \mu \mathrm{A}$ after 10 hours. B) The empty vector control reactor produced relatively no current.

To directly test the effect poising had on LamB-5rGBP binding, a second gold surface was included in the reactor. This second gold surface was not connected to the potentiostat circuitry and hence remained un-poised throughout CA analysis. This allowed imaging of both a poised and an un-poised surface from the same reactor and the same culture conditions. After electrochemical analysis, all gold electrodes were analyzed by fluorescence microscopy to determine the effect poising had on binding of the LamB-5rGBP strain (Figure 3.7). Six images were taken of each surface and representative images were chosen including images for both the 
middle and lower portions of electrodes since the surfaces tended to vary (top images include the area of the electrode under the plastic clamp and so are not included).

The poised LamB-5rGBP electrode contained fewer cells compared to the un-poised electrode (Figure 3.7 A and B versus C and D), and cells on the poised electrode stained mainly "live." Conversely, different areas on the un-poised electrode imaged quite differently. Cells bound to the middle of the un-poised electrode stained "dead" indicating a compromised membrane, while cells at the bottom of the electrode stained mainly "live" (Figure 3.7 C and D). Due to the small volume in the reactor, the unpoised electrode was tilted slightly to keep it from touching other reactor components. Due to this placement, the lower portion of the un-poised electrode was positioned near the poised electrode. Therefore, it is possible that cells bound to this section of the unpoised reactor were able to respire due to the relative proximity of a poised acceptor; whereas, the distance may have been too great for cells located in the middle section. If true, cells on the un-poised gold surface may have contributed to the current in the LamB-5rGBP reactor (Figure 3.6A). As predicted, electrodes from the reactor containing the empty vector control harbored very few cells (Figure 3.7 E and F). It is clear from the poised electrode images in Figure 3.7 why the empty vector control produced relatively no current since few cells remained attached to the surface of the electrode, yet this still does not explain the low current levels produced by the LamB-5rGBP strain since many cells are still attached. 


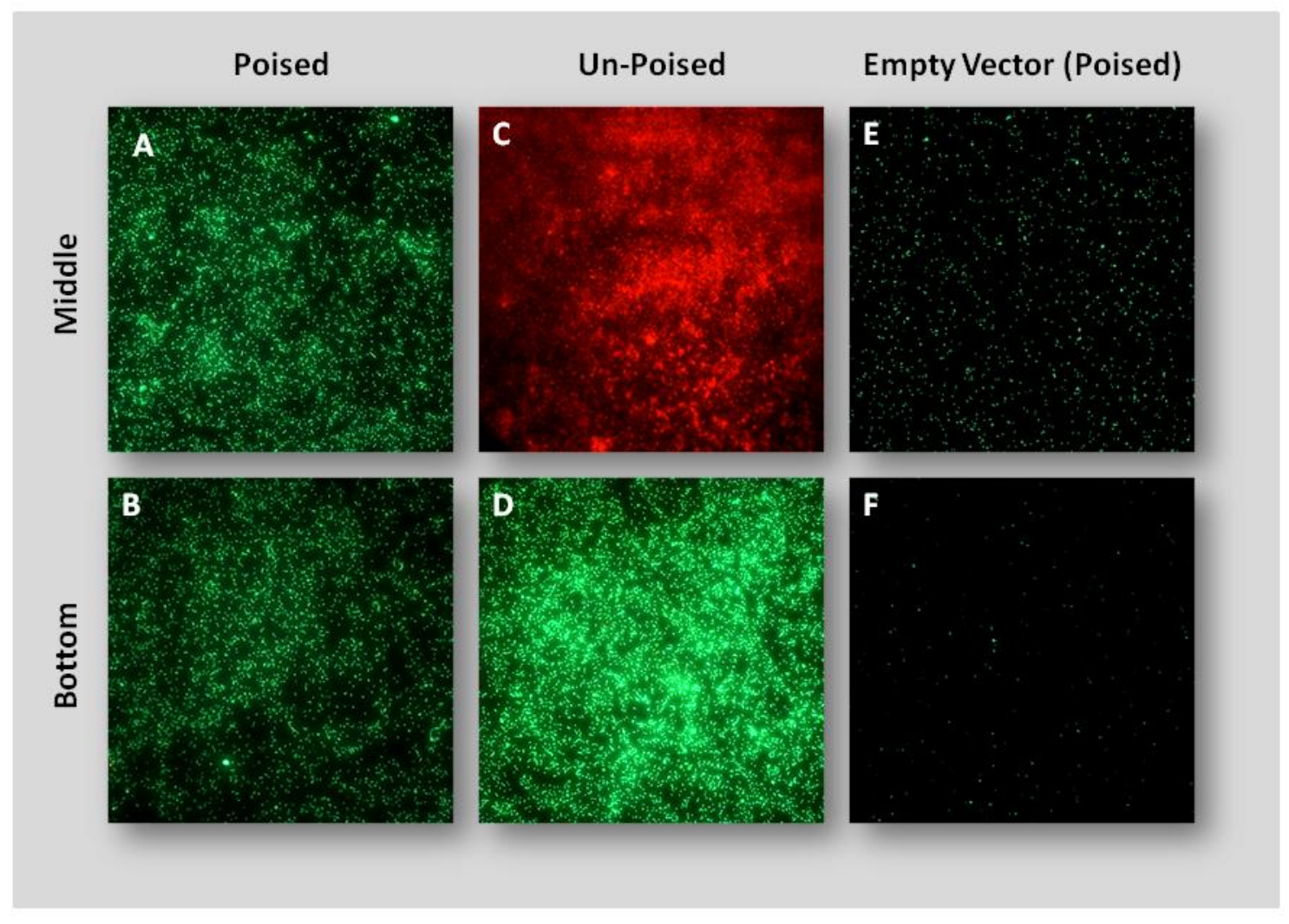

Figure 3.7. Images from poised and un-poised electrodes used for current analysis by the LamB-5rGBP expressing strain and the empty vector control. Multiple images were chosen since cell number and viability differed at various locations on certain electrodes. Images most representative of each location were chosen. The "Top" of the electrode is not included as it is the area under the clamp connecting each electrode to the reactor. The 'Poised' and 'Un-poised' columns represent images taken from the reactor containing the LamB-5rGBP expressing strain, and the last column contains images from the empty vector control reactor. The top row (A, $C$, E) consists of images representative of the middle $1 / 3$ of the electrode, while the bottom row (B, D, F) contains images typical of the bottom $1 / 3$ of the electrode.

\section{Conclusion}

Previous research using microbial fuel cells has indicated that bare gold, regardless of its highly conductive properties, is a poor material choice as an anode for Shewanella biofilms (Crittenden et al., 2006; Sun et al., 2010). To generate current in MFCs using gold electrodes, these studies had to rely on modification of the gold surface itself either through the production of self-assembled monolayers or by sputtering a gold layer atop carbon electrodes. Our study attempted to bypass the need to modify the surface and, in turn, engineered a strain capable of 
adsorption onto gold surfaces in an attempt to increase current production in gold electrode reactors.

As to the bio-incompatibility issues with Shewanella on gold, multiple hypotheses have been posed to explain the lack of coupling and include: toxicity of the surface, lack of functional groups that mediate microbe-electrode interactions, a block in transfer of electrons to the gold surface, inability to bind, and instability of mediators in redox reactions with gold. To overcome binding issues, we engineered a strain of Shewanella to express an outer membrane porin modified to display a gold-binding polypeptide, yet binding alone did not enable our cells to effectively couple with bare gold electrodes. As to the prediction of surface toxicity, our data seems to indicate otherwise. Most bound cells stain "live" on gold surfaces. Functional groups that mediate interactions between the bacterium and the electrode may be required, yet it is interesting to note that each step for electron transport via mediators works when aspects of this process are analyzed individually. Our gold-binding strain can reduce riboflavin; riboflavin can reduce gold, but when these processes are combined in reactors, only low levels of current are achieved. A block prohibiting electron transfer from cells to gold is also possible although scanning tunneling microscopy of both MtrC and OmcA has been performed on gold(111) surfaces (Wigginton et al., $2007 \mathrm{a}$ and b). As a caveat to the previous statement, both proteins had to be immobilized on the surface to be conducive to electron tunneling. Overall, the exact block inhibiting the coupling of Shewanella and gold electrodes remains unknown, but we have found that it is not due merely to inefficient binding or the instability of mediators in redox reactions with gold. 


\section{Chapter 4}

\section{Conclusions and Future Directions}

Cloning recombinant genes within plasmid carriers, an idea that revolutionized the field of molecular genetics, originated from ideas shared over a lunch of sandwiches and beer in a Hawaiian deli. Stanley Cohen of Stanford University co-organized a meeting on plasmid DNA held in Hawaii in 1972. As a suggestion from a co-organizer, Cohen invited Herbert Boyer to speak at the meeting and share data from his research utilizing restriction enzymes encoded by bacterial plasmids. As Boyer talked, Cohen, who worked with plasmids that conferred antibiotic resistance to bacterial hosts, began to outline a research plan which even he admits was a long shot at best. Cohen pitched his ideas and extended an offer to collaborate with Boyer during the now famous lunch; an offer Boyer initially declined. Building on the seminal recombinant DNA work by Paul Berg, Boyer and Cohen generated the first recombinant plasmids and were the first to express both foreign bacterial and eukaryotic genes within plasmid vectors.

The progression of genetic engineering technologies along with improvements in DNA sequencing now allow us to engineer microorganisms to produce specific products or perform desired tasks. In the current study, cell surface display, a recombinant DNA technique, was utilized to express a modified E. coli outer membrane protein altered to incorporate a goldbinding polypeptide in order to direct adsorption of Shewanella onto gold surfaces. Previous research attempting to use gold anodes in MFCs found that Shewanella was unable to use gold as a final electron acceptor despite its high conductivity unless the surface itself was modified. To encourage microbe-electrode interactions and increase current production in gold electrode reactors without relying on surface modification, the basis of my project was to engineer a strain of Shewanella able to adsorb onto gold surfaces via the heterologous expression of LamB-5rGBP.

The fact that Shewanella is unable to couple with gold is not entirely surprising since this surface differs greatly both at the molecular and chemical levels compared to surfaces Shewanella species encounter naturally. Theories to explain this bio-incompatibility have included: inability to bind, toxicity of the surface, lack of functional groups that mediate microbe-electrode interactions, a block in electron transfer between the microbe and the surface, and instability of mediators in redox reactions with gold. Binding issues were overcome by directing adhesion onto 
gold(111) surfaces through the expression of LamB-5rGBP, yet the expression of this heterologous protein also led to the disruption of certain outer membrane components important in metal reduction. Displacement of these proteins decreased the rates at which Shewanella was able to reduce both insoluble iron and riboflavin.

Other coupling issues involve interactions at the microbe-electrode interface. As to toxicity of the gold surface, our results seem to indicate otherwise. The majority of cells bound to gold stained "live" indicating that binding did not compromise membrane integrity. As to the stability of outer membrane proteins, strong adsorption onto gold has been shown to denature certain cytochromes, yet scanning tunneling microscopy and tunneling spectroscopy of MtrC and OmcA have been performed by specifically immobilizing each onto the gold(111) surface (Wigginton et al., $2007 \mathrm{a}$ and b). Wigginton's research may also negate the idea of a block in electron transfer occurring between Shewanella and gold. It is interesting that relatively no electron transfer occurs between Shewanella and gold, yet this bacterium can reduce extracellular semiconductive metal oxides. Our data also indicates that the redox-active mediator riboflavin is stable and quite efficient during electron transfer reactions with gold. To date, efficient coupling of Shewanella and gold electrodes has only occurred through the modification of gold surfaces to include functional groups similar to those found in humic substances.

While the expression of LamB-5rGBP did effectively direct attachment to gold surfaces, it did not lead to much increase in current for reactors containing unmodified gold electrodes. Therefore, it can be concluded that the inability of Shewanella to utilize bare gold electrodes is not due merely to its inability to bind. Further conclusions as to why coupling is not seen on bare gold are not as easily summed up and are compounded by the fact that each step involved electron transfer from Shewanella to gold works when analyzed individually. Our engineered strain adheres to gold surfaces; this strain can reduce riboflavin, and riboflavin can reduce gold surfaces. Yet, when all three steps are combined in anaerobic bioreactors, very little current is generated. This research successfully led to the engineering of a strain specific for binding gold surfaces, but ends with many questions yet unanswered as to the interactions between Shewanella and gold.

Although this strategy did not increase current production in bioreactors, the ability to specifically immobilize cells on defined surfaces is potentially useful in many other applications. 
Gold surfaces can be fabricated to exhibit highly defined topologies and shapes, meaning that the overall control for designing where Shewanella "sticks" on surfaces is practically limitless.

Technology like this could be used to generate whole-cell biosensors and also engineer specific multispecies biofilms. Incorporation of additional surface-displayed peptides is also possible, meaning that specific enzymatic activities can be spatially organized within highly complex systems. Surface binding also has the potential to aid in bioremediation applications. Shewanella species are able to respire certain toxic metals such as uranium, technetium, and cobalt, whose solubility in ground water and soils is dependent upon their oxidation state (Hau and Gralnick, 2007). U(VI) is soluble whereas U(IV) precipitates out of solution; therefore, by immobilizing Shewanella on a surface, these toxic metals could be captured as well. 


\section{References}

Boos, W., and H. Shuman. 1998. Maltose/maltodextrin system of Escherichia coli: transport, metabolism, and regulation. Microbiol. Mol. Biol. Rev. 62:204-229.

Boulain, J. C., Charbit, A., and M. Hofnung. 1986. Mutagenesis by random linker insertion into the lamB gene of Escherichia coli K12. Mol. Gen. Genet. 205:339-348.

Brown, S. 1997. Metal-recognition by repeating polypeptides. Nat. Biotechnol.15:269-272.

Carabineiro, S. A. C., and B. E. Nieuwenhuys. 2009. Adsorption of small molecules on gold single crystal surfaces. Gold Bull. 42:288-301.

Charbit, A., Boulain, J. C., Ryter, A., and M. Hofnung. 1986. Probing the topology of a bacterial membrane protein by genetic insertion of a foreign epitope; expression at the cell surface. E.M.B.O. J. 5:3029-3037.

Chen, X. X., Ferrigno, R., Yang, J., and G. A. Whitesides. 2002. Redox properties of cytochrome $c$ adsorbed on self-assembled monolayers: a probe for protein conformation and orientation. Langmuir. 18:7009-7015.

Clement, J. M., and M. Hofnung. 1981. Gene sequence of the lambda receptor, an outer membrane protein of Escherichia coli K12. Cell. 27:507-514.

Coursolle, D., Baron, D. B., Bond, D. R., and J. A. Gralnick. 2010. The Mtr respiratory pathway is essential for reducing flavins and electrodes in Shewanella oneidensis. J. Bacteriol. 192:467-474.

Coursolle, D., and J. A. Gralnick. 2010. Modularity of the Mtr respiratory pathway of Shewanella oneidensis strain MR-1. Mol. Microbiol. 77:995-1008.

Crittenden, S. R., Sund, C. J., and J. J. Sumner. 2006. Mediating electron transfer from bacteria to a gold electrode via a self-assembled monolayer. Langmuir. 22:9473-9476.

Debarbouille, M., Shuman, H. A., Silhavy, T. J., and M. Schwartz. 1978. Dominant constitutive mutations in malT, the positive regulator gene of the maltose regulon in Escherichia coli. J. Mol. Biol. 124:359-371.

Fenter, P., Eberhardt, A., and P. Eisenberger. 1994. Self-assembly of n-alkyl thiols as disulfides on $\mathrm{Au}(111)$. Science. 266:1216-1218.

Firer-Sherwood, M., Pulcu, G. S., and S. J. Elliott. 2008. Electrochemical interrogations of the Mtr cytochromes from Shewanella: opening a potential window. J. Biol. Inorg. Chem. 13:849854. 
Fredrickson, J. K., Romine, M. F., Beliaev, A. S., Auchtung, J. M., Driscoll, M. E., Gardner, T., Nealson, K. H., Osterman, A. L., Pinchuk, G., Reed, J. L., Rodionov, D. A., Rodrigues, J. L., Saffarini, D. A., Serres, M. S., Spormann, A. M., Zhulin, I. B., and J. M. Tiedje. 2008. Towards environmental systems biology of Shewanella. Nat. Rev. Microbiol. 6:592-603.

Freudl, R., Macintyre, S., Degen, M., and U. Henning. 1986. Cell surface exposure of the outer membrane protein OmpA of Escherichia coli K-12. J. Mol. Biol. 188:491-494.

Ghisla, S., Massey, V., Lhoste, J. M., and S. G. Mayhew. 1974. Fluorescence and optical characteristics of reduced flavines and flavoproteins. Biochem. 13:589-597.

Gilson, E., Rousset, J. P., Charbit, A., Perrin, D., and M. Hofnung. 1986. malM, a new gene of the maltose regulon in Escherichia coli K12. I. malM is the last gene of the malK-lamB operon and encodes a periplasmic protein. J. Mol. Biol. 191:303-311.

Gorby, Y. A., Yanina, S., McLean, J. S., Rosso, K. M., Moyles, D., Dohnalkova, A., Beveridge, T. J., Chang, I. S., Kim, B. H., Kim, K. S., Culley, D. E., Reed, S. B., Romine, M. F. Saffarini, D. A., Hill, E. A., Shi, L., Elias, D. A., Kennedy, D. W., Pinchuk, G., Watanabe, K., Ishii, S., Logan, B., Nealson, K. H., and J. K. Fredrickson. 2006. Electrically conductive bacterial nanowires produced by Shewanella oneidensis strain MR-1 and other microorganisms. Proc. Natl. Acad. Sci. U. S. A. 103:11358-11363.

Gralnick, J. A., and D. K. Newman. 2007. Extracellular respiration. Mol. Microbiol. 65:1-11

Hartshorne, R. S., Reardon, C. L., Ross, D., Nuester, J., Clarke, T. A., Gates, A. J., Mills, P. C., Fredrickson, J. K., Zachara, J. M., Shi, L., Beliaev, A. S., Marshall, M. J., Tien, M., Brantley, S., Butt, J. N., and D. J. Richardson. 2009. Characterization of an electron conduit between bacteria and the extracellular environment. Proc. Natl. Acad. Sci. U. S. A. 106:2216922174.

Hau, H. H., Gilbert, A., Coursolle, D., and J. A. Gralnick. 2008. Mechanism and consequences of anaerobic respiration of cobalt by Shewanella oneidensis strain MR-1. Appl. Environ. Microbiol. 74:6880-6886.

Hau, H. H., and J. A. Gralnick. 2007. Ecology and biotechnology of the genus Shewanella. Annu. Rev. Microbiol. 61:237-258.

Heidelberg, J. F., Paulsen, I. T., Nelson, K. E., Gaidos, E. J., Nelson, W. C., Read, T. D., Eisen, J. A., Seshadri, R., Ward, N., Methe, B., Clayton, R. A., Meyer, T., Tsapin, A., Scott, J., Beanan, M., Brinkac, L., Daugherty, S., DeBoy, R. T., Dodson, R. J., Durkin, A. S., Haft, D. H., Kolonay, J. F., Madupu, R., Peterson, J. D., Umayam, L. A., White, O., Wolf, A. M., Vamathevan, J., Weidman, J., Impraim, M., Lee, K., Berry, K., Lee, C., Mueller, J., Khouri, H., Gill, J., Utterback. T. R., McDonald, L. A., Feldblyum, T. V., Smith, H. O., Venter, J. C., Nealson, K. H., and C. M. Fraser. 2002. Genome sequence of the dissimilatory metal ion-reducing bacterium Shewanella oneidensis. Nat. Biotechnol. 20:1118-1123. 
Heinz, H., Farmer, B. L., Pandey, R. B., Slocik, J. M., Patnaik, S. S., Pachter, R., and R. R. Naik. 2009. Nature of molecular interactions of peptides with gold, palladium, and $\mathrm{Pd}-\mathrm{Au}$ bimetal surfaces in aqueous solution. J. Am. Chem. Soc. 131:9704-9714.

Hoefling, M., Iori, F., Corni, S., and K. Gottschalk. 2010. Interaction of amino acids with the $\mathrm{Au}(111)$ surface: adsorption free energies from molecular dynamics simulations. Langmuir. 26:8347-8351.

Hoefling, M., Iori, F., Corni, S., and K. Gottschalk. 2010. The conformations of amino acids on a gold(111) surface. ChemPhysChem. 11:1763-1767.

Hor, L. I., and H. A. Shuman. 1993. Genetic analysis of periplasmic binding protein dependent transport in Escherichia coli: each lobe of maltose-binding protein interacts with a different subunit of the $\mathrm{MalFGK}_{2}$ membrane transport complex. J. Mol. Biol. 233:659-670.

Kim, H. J., Park, H. S., Hyun, M. S., Chang, I.S., Kim, M., and B. H. Kim. A mediator-less microbial fuel cell using a metal reducing bacterium, Shewanella putrefaciens. Enzyme Microb. Tech. 30:145-152.

Lee, S. Y., Choi, J. H., and Z. H. Xu. 2003. Microbial cell-surface display. Trends Biotechnol. 21:45-52.

Lies, D. P., Hernandez, M. E., Kappler, A., Mielke, R. E., Gralnick, J. A., and D. K.

Newman. 2005. Shewanella oneidensis MR-1 uses overlapping pathways for iron reduction at a distance and by direct contact under conditions relevant for biofilms. Appl. Environ. Microbiol. 71:4414-4426.

Liu, Y., Kim, H., Franklin, R., and D. R. Bond. 2010. Gold line array electrodes increase substrate affinity and current density of electricity-producing G. sulfurreducens biofilms. Energy Environ. Sci. 3:1782-1788.

Lovley, D. R. 2002. Dissimilatory metal reduction: from early life to bioremediation. A. S. M. News. 68:231-237.

Lovley, D. R., Coates, J. D., Blunt-Harris, E. L., Phillips, E. J. P., Woodward, J. C. 1996. Humic substances as electron acceptors for microbial respiration. Nature. 382:445-448.

Marshall, M. J., Beliaev, A. S., Dohnalkova, A. C., Kennedy, D. W., Shi, L., Wang, Z., Boyanov, M. I., Lai, B., Kemner, K. M., McLean, J. S., Reed, S. B., Culley, D. E., Bailey, V. L., Simonson, C. J., Saffarini, D. A., Romine, M. F., Zachara, J. M., and J. K. Fredrickson. $c$-Type cytochrome-dependent formation of U(IV) nanoparticles by Shewanella oneidensis. P. L. O. S. Bio. 4:1324-1333.

Marsili, E., Baron, D. B., Shikhare, I. D., Coursolle, D., Gralnick, J. A., and D. R. Bond. 2008. Shewanella secretes flavins that mediate extracellular electron transfer. Proc. Natl. Acad. Sci. U. S. A. 105:3968-3973. 
Myers, C. R., and J. M. Myers. 2002. MtrB is required for proper incorporation of the cytochromes OmcA and OmcB into the outer membrane of Shewanella putrefaciens MR-1. Appl. Environ. Microbiol. 68:5585-5594.

Nealson, K. H., and J. Scott. 2006. Ecophysiology of the Genus Shewanella. In: The Prokaryotes. Vol 6. 3rd ed. Springer New York; 1133-1151.

Newton, S. M. C., Klebba, P. E., Michel, V., Hofnung, M., and A. Charbit. 1996. Topology of the membrane protein LamB by epitope tagging and a comparison with the X-ray model. J. Bacteriol. 178:3447-3456.

Pitts, K. E., Dobbin, P. S., Reyes-Ramirez, F., Thomson, A. J., Richardson, D. J., and H. E. Seward. 2003. Characterization of the Shewanella oneidensis MR-1 decaheme cytochrome MtrA. J. Biol. Chem. 278:27758-27765.

Randall-Hazelbauer, L., and M. Schwartz. 1973. Isolation of the bacteriophage lambda receptor from Escherichia coli. J. Bacteriol. 116:1436-1446.

Richter, H., McCarthy, K., Nevin, K. P., Johnson, J. P., Rotello, V. M., and D. R. Lovley. 2008. Electricity generation by Geobacter sulfurreducens attached to gold electrodes. Langmuir. 24:4376-4379.

Ross, D. E., Ruebush, S. S., Brantley, S. L., Hartshorne, R. S., Clarke, T. A., Richardson, D. J., and M. Tien. 2007. Characterization of protein-protein interactions involved in iron reduction by Shewanella oneidensis MR-1. Appl. Environ. Microbiol. 73:5797-5808.

Rutherford, N., and M. Mourez. 2006. Surface display of proteins by Gram-negative bacterial autotransporters. Microb. Cell Fact. 5:22-36.

Saltikov, C. W., and D. K. Newman. 2003. Genetic identification of a respiratory arsenate reductase. Proc. Natl. Acad. Sci. U. S. A. 100:10983-10988.

Sarikaya, M., Tamerler, C., Jen, A. K. Y., Schulten, K., and F. Baneyx. 2003. Molecular biomimetics: nanotechnology through biology. Nat. Mater. 2:577-585.

Schirmer, T., Keller, T. A., Wang, Y. F., and J. P. Rosenbusch. 1995. Structural basis for sugar translocation through maltoporin channels at 3.1 Angstrom resolution. Science. 267:512514.

Schouteden, K., Lievens, P., and C. Van Haesendonck. 2009. Fourier-transform scanning tunneling microscopy investigation of the energy versus wave vector dispersion of electrons at the Au(111) surface. Phys. Review B. 79:195409-1-7.

Schwalb, C., Chapman, S. K., and G. A. Reid. 2003. The tetraheme cytochrome CymA is required for anaerobic respiration with dimethyl sulfoxide and nitrite in Shewanella oneidensis. Biochemistry. 42:9491-9497. 
Shi, L., Squier, T. C., Zachara, J. M., and J. K. Fredrickson. 2007. Respiration of metal (hydr)oxides by Shewanella and Geobacter: a key role for multihaem $c$-type cytochromes. Mol. Microbiol. 65:12-20.

Smith, G. P. 1985. Filamentous fusion phage: novel expression vectors that display cloned antigens on the virion surface. Science. 228:1315-1317.

Stooky, L. L. 1970. Ferrozine - a new spectrophotometric reagent for iron. Anal. Chem. 42: 779781.

Sukchawalit, R., Vattanaviboon, P., Sallabhan, R., and S. Mongkolsuk. 1999. Construction and characterization of regulated L-arabinose-inducible broad host range expression vectors in Xanthomonas. F.E.M.S. Microbiol. Lett. 181:217-223.

Sun, M., Zhang, F., Tong, Z., Sheng, G., Chen, Y., Zhao, Y., Chen, Y., Zhou, S., Liu, G., Tian, Y., and H. Yu. 2010. A gold-sputtered carbon paper as an anode for improved electricity generation from a microbial fuel cell inoculated with Shewanella oneidensis MR-1. Biosens. Bioelectron. 26:338-343.

Szmelcman, S., and M. Hofnung. 1975. Maltose transport in Escherichia coli K-12: involvement of bacteriophage lambda receptor. J. Bacteriol. 124:112-118.

Tamerler, C., Duman, M., Oren, E. E., Gungormus, M., Xiong, X., Kacar, T., Parviz, B. A., and M. Sarikaya. 2006. Materials specificity and directed assembly of a gold-binding peptide. Small. 2:1372-1378.

Thomas, P. E., Ryan, D., and Levin, W. 1976. An improved staining procedure for the detection of the peroxidase activity of cytochrome P-450 on sodium dodecyl sulfate polyacrylamide gels. Anal. Biochem. 75: 168-176.

Ulman, A. 1996. Formation and structure of self-assembled monolayers. Chem. Rev. 96:15331554 .

Venkateswaran, K., Moser, D. P., Dollhopf, M. E., Lies, D. P., Saffarini, D. A., MacGregor, B. J., Ringelberg, D. B., White, D. C. Nishijima, M., Sano, H., Burghardt, J., Stackebrandt, E., and K. H. Nealson. 1999. Polyphasic taxonomy of the genus Shewanella and description of Shewanella oneidensis sp. nov. Int. J. Syst. Bacteriol. 49:705-724.

Verde, A. V., Acres, J. M., and J. K. Maranas. 2009. Investigating the specificity of peptide adsorption on gold using molecular dynamics simulations. Biomacromolecules. 10:2118-2128.

von Canstein, H., Ogawa, J., Shimizu, S., and J. R. Lloyd. 2008. Secretion of flavins by Shewanella species and their role in extracellular electron transfer. Appl. Environ. Microbiol. 74:615-623. 
Wang, Y. 2002. The function of OmpA in Escherichia coli. Biochem. and Biophys. Research Comm. 292:396-401.

Wigginton, N. S., Rosso, K. M., and M. F. Hochella Jr. 2007. Mechanisms of electron transfer in two decaheme cytochromes from a metal-reducing bacterium. J. Phys. Chem. B. 111:1285712864.

Wigginton, N. S., Rosso, K. M., Lower, B. H., Shi, L., and M. F. Hochella Jr. 2007. Electron tunneling properties of outer-membrane decaheme cytochromes from Shewanella oneidensis. Geochim. Cosmochim. Acta. 71:543-555. 